\title{
High average current electron guns for high-power free electron lasers
}

\author{
Phillip Sprangle, Joseph Peñano, Bahman Hafizi, ${ }^{*}$ Daniel Gordon, Steven Gold, Antonio Ting, and Chad Mitchell \\ Plasma Physics Division, Naval Research Laboratory, Washington, D.C. 20375, USA
}

(Received 14 June 2010; published 14 February 2011)

\begin{abstract}
High average power free-electron lasers (FELs) require high average current electron injectors capable of generating high quality, short duration electron bunches with a repetition rate equal to the frequency of the rf linac. In this paper we propose, analyze, and simulate an rf-gated, gridded thermionic electron gun for use in high average power FELs. Thermionic cathodes can provide the necessary high current, have long lifetimes, and require modest vacuums. In the proposed configuration the rf-gated grid is modulated at the fundamental and 3rd harmonic of the linac frequency. The addition of the 3rd harmonic on the grid results in shorter electron bunches. In this configuration, every rf bucket of the linac accelerating field contains an electron bunch. Particle-in-cell simulations indicate that this approach can provide the necessary charge per bunch, bunch duration, longitudinal and transverse emittance, and repetition rate for high average power FELs operating in the IR regime.
\end{abstract}

DOI: 10.1103/PhysRevSTAB.14.020702

PACS numbers: 41.60.Cr, 07.77.Ka

\section{INTRODUCTION}

High average power free-electron lasers (FELs) [1-9] require a train of short duration, high repetition rate, and high peak current electron bunches having high quality and high average current [1]. These requirements place stringent constraints on the electron gun [2-4].

Figure 1 shows a schematic of an FEL injector for high average power operation. For IR FELs the typical electron beam parameters associated with the various stages in the injector are indicated. The injector should produce an electron pulse train with short $(\sim 10$ psec rms $)$ bunches at a repetition frequency of $\sim 700 \mathrm{MHz}$, with energies of $\sim 5-8 \mathrm{MeV}$, charge per bunch of $\sim 1 \mathrm{nC}$, normalized transverse emittance of $\varepsilon_{n, \perp}<15 \mathrm{~mm}$ mrad, and a bunch radius of $R_{b} \sim 0.3 \mathrm{~mm}$. Before entering the FEL wiggler the electron bunches are accelerated in an energy recovery linac (ERL) to $\sim 80 \mathrm{MeV}$ with a total fractional longitudinal energy spread $\Delta E_{\text {total }} / E<0.5 \%$, peak bunch current $I_{b} \sim 400 \mathrm{~A}$, and an average current of $\sim 1 \mathrm{~A}$, while preserving the beam emittance and radius. Assuming a Gaussian longitudinal charge profile, the charge per bunch entering the wiggler is $q N_{b} \approx \sqrt{2 \pi} I_{b} \tau_{b} \sim 1 \mathrm{nC}$, where $\tau_{b} \sim 1 \mathrm{psec}$ is the rms bunch duration (rms duration is related to the FWHM by $\tau_{b}=\tau_{\mathrm{FWHM}} / 2.35$ ). The beam quality requirements for high-power IR FEL operation are discussed in more detail in Appendix A. In addition, while high average power FELs require high wall-plug efficiencies, the injector efficiency does not play a significant role in determining the overall wall-plug efficiency [9].

Photocathode guns are capable of generating short, highquality, and high-charge electron bunches and are employed in the majority of FELs [1-4,10-13]. However, thermal issues, short lifetimes, and drive-laser average-

*Present address: Icarus Research, Inc., Bethesda, MD. power requirements currently present limitations when operated at high average powers.

Thermionic guns (Appendix B) have demonstrated long lifetime operation and high average currents. Several FELs have used thermionic guns such as the Boeing [14], Stanford University [15,16], Novosibirsk [17], SPring-8 $[18,19]$, and FELIX [20] FELs. These guns cannot be used for high average power IR FELs because of their low repetition rate. The first three examples were gridded guns, whose microbunch repetition rates were limited because the grids were not directly driven with rf. The SPring-8 FEL used a beam chopper to extract a single electron bunch from a nongridded electron gun driven by a high voltage pulse from a conventional modulator. The FELIX FEL operated for nearly 20 years with a gridded thermionic gun directly driven by a $1 \mathrm{GHz}$ rf source with a DC bias, but without the use of harmonics. Here, we propose a new, harmonically gated injector configuration based on a thermionic gun that can satisfy the requirements for a high average power IR FEL.

This paper proposes the use of rf-gated, harmonically driven, gridded thermionic electron guns for high average power IR FELs. In the proposed configuration, the rf-gated grid is modulated at the fundamental and 3rd harmonic of the rf linac frequency, e.g., $\sim 700 \mathrm{MHz}$ and $\sim 2.1 \mathrm{GHz}$. Simulations indicate that the required electron beam quality, pulse duration, and average current for high average power FEL operation can be achieved with this configuration. In addition, the heat radiated by the thermionic gun in the proposed design is relatively small, and hence we believe it can be used with either normal-conducting or superconducting prebooster rf cavities $[3,4,10]$.

In Sec. II we discuss the characteristics of the rf-gated, gridded thermionic gun and the use of rf harmonics to generate short electron bunches. Section II also contains an analysis and estimates of the bunch emittance due to the 


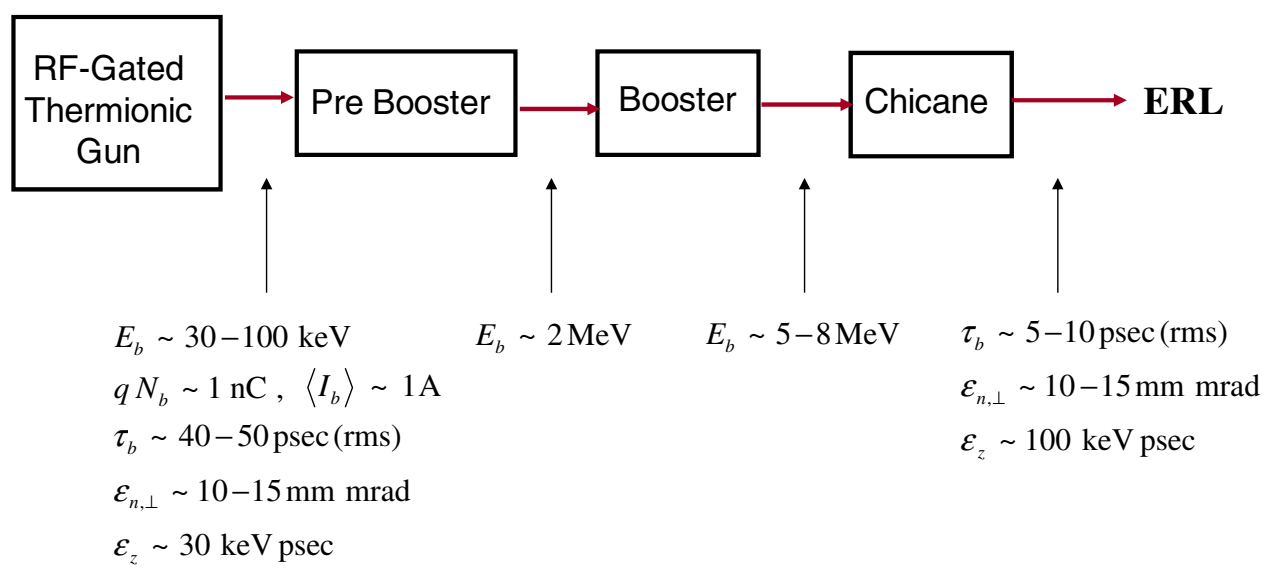

FIG. 1. Schematic of injector for an FEL employing an energy recovery linac (ERL). The source of electrons is an rf-gated, gridded thermionic gun. Electrons are accelerated in booster cavities and bunched in a magnetic chicane before entering the ERL. Typical electron beam parameters associated with the stages are indicated in the figure. Additional bunching is achieved in the ERL and a chicane just before the wiggler.

grid. Section III presents the results of particle-in-cell (PIC) simulations of thermionic injector operation using the TURBOWAVE [21] and PARMELA [22] codes. Section IV summarizes our theoretical and numerical results. A fully functioning prototype may require that the bunch emittance be lowered by reshaping the cathode and anode. Appendices A, B, and C present additional details of the thermionic gun.

\section{RF-GATED, GRIDDED THERMIONIC GUN}

Figure 2 shows a schematic of the rf-gated, gridded thermionic cathode configuration. The grid consists of an array of concentric wires, close to the cathode, that is modulated by rf fields. The grid has a negative dc bias with respect to the cathode, in addition to the rf fields at the fundamental and 3rd harmonic of the linac frequency. Since the grid is modulated at the fundamental linac frequency, every accelerating rf bucket is filled. The addition of the 3rd harmonic provides shorter electron bunches. The typical parameters of the gun are shown in Table I. The key characteristics of the gun are discussed below.

\section{A. Thermionic cathode}

Thermionic cathodes can provide the necessary high average current for high average power FELs [1419,23,24]. They operate at moderate vacuums of $10^{-8}-10^{-7}$ Torr [25] and can have long lifetimes ( $>10000$ hours) delivering over $10^{8}$ coulombs [23]. As an example, for a cathode current density of $J=$ $10 \mathrm{~A} / \mathrm{cm}^{2}$, the charge per bunch is $q N_{b}=2.35 \pi R_{b}^{2} J \tau_{b} \approx$ $1 \mathrm{nC}$ for a bunch radius $R_{b}=0.5 \mathrm{~cm}$ and $\mathrm{rms}$ bunch duration $\tau_{b}=54 \mathrm{psec}$. These parameters are readily achieved with conventional thermionic cathodes. One candidate for this application is a dispenser cathode which can generate current densities of $\sim 10 \mathrm{~A} / \mathrm{cm}^{2}$, while more advanced cathodes (with controlled porosity) can generate up to $\sim 100 \mathrm{~A} / \mathrm{cm}^{2}$. Single-crystal cathodes such as lanthanum hexaboride and cerium hexaboride are robust and can generate up to $\sim 100 \mathrm{~A} / \mathrm{cm}^{2}$, but require high temperature $[18,19]$. Dispenser cathodes employing scandate oxides have low work functions $(\sim 1.5 \mathrm{eV})$, high current densities $\left(>100 \mathrm{~A} / \mathrm{cm}^{2}\right)$, and long lifetimes $(>1000 \mathrm{hr})$, but may have poor reproducibility and nonuniform emission [23].

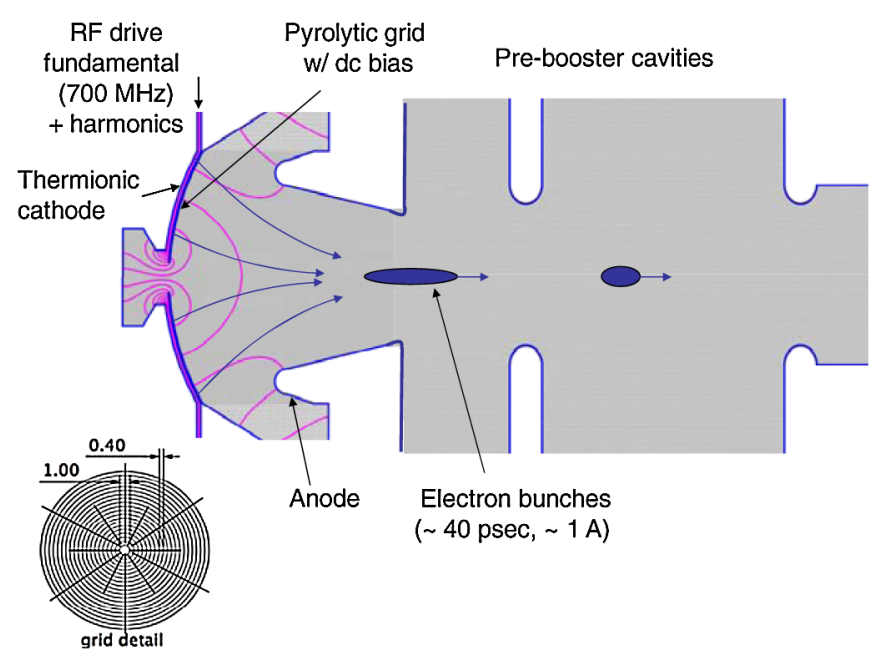

FIG. 2. Schematic diagram of rf-gated thermionic gun for high average current FEL operation. The rf-gated, gridded thermionic gun consists of a dispenser or single-crystal $\mathrm{CeB}_{6}$ cathode and pyrolytic graphite grid. The grid consists of a series of concentric rings and is biased negatively and driven positive by the $\mathrm{rf}$ relative to the cathode. Typical grid dimensions are indicated in the figure in $\mathrm{mm}$. The potential on the grid is modulated at the fundamental $(\sim 700 \mathrm{MHz})$ and 3rd harmonic $(2.1 \mathrm{GHz})$ of the linac frequency to produce short $(\sim 40 \mathrm{psec} \mathrm{rms})$, high-charge $(\sim 1 \mathrm{nC})$ electron bunches. The rf field of the fundamental loads every bucket in the linac with an electron bunch. The electron energy exiting the prebooster is $\sim 2 \mathrm{MeV}$. The grid voltage is negative and typically in the range of 100-300 V. 
TABLE I. Typical range of parameters in an rf-gated, gridded thermionic gun in which a conventional dispenser cathode is employed.

\begin{tabular}{ll}
\hline \hline Cathode-grid gap, $z_{G}$ & $250 \mu \mathrm{m}$ \\
Cathode-anode gap, $z_{A}$ & $1-3 \mathrm{~cm}$ \\
Grid wire radius, $r_{o}$ & $30 \mu \mathrm{m}$ \\
Grid wire separation, $2 a$ & $400-500 \mu \mathrm{m}$ \\
Cathode radius, $R_{c}$ & $0.5-1.5 \mathrm{~cm}$ \\
Cathode-grid voltage, $\Phi_{G}$ & $100-300 \mathrm{~V}$ \\
Cathode-anode voltage, $\Phi_{A}$ & $30-50 \mathrm{kV}$ \\
rf grid modulation frequency, $f_{\mathrm{rf}}$ & $500 \mathrm{MHz}-2.5 \mathrm{GHz}$ \\
Cathode current density, $J$ & $10-15 \mathrm{~A} / \mathrm{cm}^{2}$ \\
Cathode temperature, $T_{c}$ & $1200-1400 \mathrm{~K}$ \\
Cathode work function, $\Phi_{\mathrm{WF}}$ & $1.6-2.1 \mathrm{eV}$ \\
Vacuum level & $10^{-7}-10^{-8} \mathrm{Torr}$ \\
\hline \hline
\end{tabular}

The current-voltage characteristics of thermionic cathodes are determined by the material, temperature as well as the external and space-charge fields. In the steadystate, 1D limit, the space-charge limited current density is given by the Child-Langmuir (CL) relationship [23,2628], $J_{\mathrm{CL}}\left[\mathrm{A} / \mathrm{cm}^{2}\right]=2.33 \times 10^{-6} \Phi^{3 / 2}[\mathrm{~V}] / d^{2}[\mathrm{~cm}]$, where $\Phi \geq 0$ is the voltage difference between the two conducting planes and $d$ is the separation.

In the temperature-limited regime, i.e., the RichardsonDushman-Schottky (RDS) limit, the current density is given by $[29,30]: J_{\mathrm{RDS}}\left[\mathrm{A} / \mathrm{cm}^{2}\right]=A_{o} T_{c}^{2} \exp \left\{-11605 \Phi_{\mathrm{WF}} /\right.$ $\left.T_{c}\right\} \exp \left\{4.4(\Phi[\mathrm{V}] / d[\mathrm{~cm}])^{1 / 2} / T_{c}\right\}$, where $A_{o}=120 \mathrm{~A} /$ $\left(\mathrm{cm}^{2} \mathrm{~K}^{2}\right), \Phi_{\mathrm{WF}}$ is the work function $(\mathrm{eV})$ of the cathode and $T_{c}$ is the temperature (K). The above value for $A_{o}$ is the theoretically derived value while the experimentally measured value for $\mathrm{CeB}_{6}$ indicates that $A_{o} \approx 20 \mathrm{~A} /\left(\mathrm{cm}^{2} \mathrm{~K}^{2}\right)$ [18].

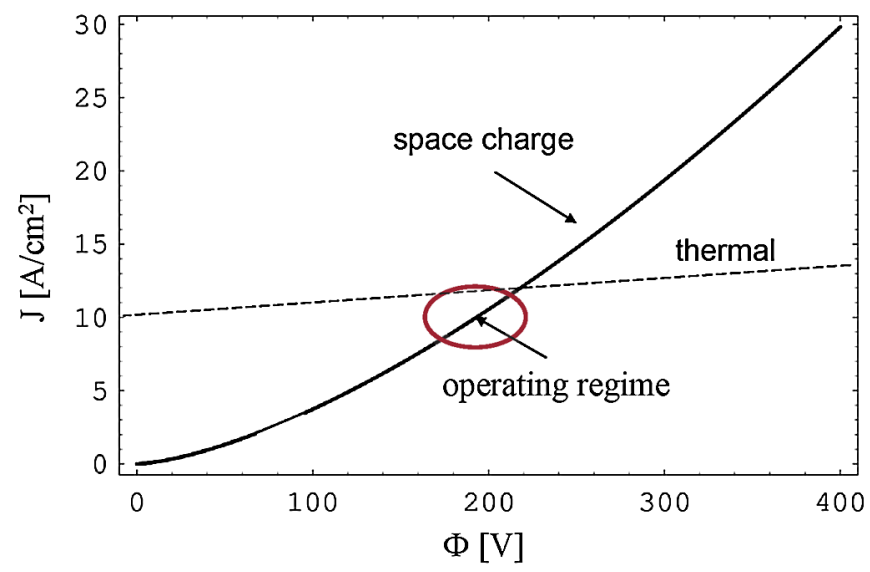

FIG. 3. Current density-voltage characteristics, $J$ vs $\Phi$. The solid curve is the space-charge limit (Child-Langmuir) and the dashed curve is the thermal limit (Richardson-DushmanSchottky). The characteristics are for the case with gap spacing $d=250 \mu \mathrm{m}$, work function $\Phi_{\mathrm{WF}}=1.9 \mathrm{eV}$, and cathode temperature $T_{c}=1300 \mathrm{~K}$. The optimum operating region is near the intersection of the two limits where the current density is not a sensitive function of the cathode temperature.
The Child-Langmuir and the Richardson-DushmanSchottky regimes are plotted in Fig. 3 for $d=250 \mu \mathrm{m}$, $\Phi_{\mathrm{WF}}=1.9 \mathrm{eV}, T_{c}=1300 \mathrm{~K}$, and $A_{o}=120 \mathrm{~A} /\left(\mathrm{cm}^{2} \mathrm{~K}^{2}\right)$. The optimum operating regime for the rf-gated gun is near the intersection of the space-charge and thermal limits where the current density is not sensitive to fluctuations in the cathode temperature as shown in Fig. 3.

There is a third emission regime in which a high surface electric field $E_{S} \sim 10^{7}-10^{8} \mathrm{~V} / \mathrm{cm}$ is employed to extract electrons by tunneling. This field emission regime is particularly effective when the cathode consists of sharp tips. In this regime the current density is given by the Fowler-Nordheim $(\mathrm{FN})$ relation $[23,31], J_{\mathrm{FN}}\left[\mathrm{A} / \mathrm{cm}^{2}\right]=$ $A_{\mathrm{FN}} E_{S}^{2} \exp \left(-B_{\mathrm{FN}} / E_{S}+9.8 / \sqrt{\Phi_{\mathrm{WF}}}\right)$, where $A_{\mathrm{FN}}\left[\mathrm{A} / \mathrm{V}^{2}\right] \approx$ $1.4 \times 10^{-6} / \Phi_{\mathrm{WF}}, B_{\mathrm{FN}}[\mathrm{V} / \mathrm{cm}] \approx 6.5 \times 10^{7} \Phi_{\mathrm{WF}}^{3 / 2}$, and $E_{S}$ is in units of $\mathrm{V} / \mathrm{cm}$. Field emitting cathodes are also being considered for high average current guns for FELs [32].

\section{B. rf-gated grid}

The actual grid configuration consists of a series of concentric rings and radial spokes located close to the cathode surface as shown in Fig. 2. However, for the purpose of the following analysis, the rings are modeled by an array of parallel wires as shown in Fig. 4 and the rf modulation is treated electrostatically.

The potential on the grid wires is $\Phi_{G}$, the potential difference between the cathode and anode is $\Phi_{A}$. The grid wires are parallel to the $y$ axis, separated by a distance $2 a$ and located at $z=z_{G}$. The radius of the grid wires is taken to be small, $r_{o} \ll a, z_{G}$. The potential in the cathodegrid-anode region is given by (Appendix $\mathrm{C}$ )

$$
\begin{aligned}
\Phi(x, z)= & -\sum_{n= \pm 1, \pm 3,}\left[\Phi_{O} \ln \frac{G_{n}(x, z)}{F_{n}(x, z)}+\Phi_{A} \frac{r_{o}^{2}\left(z-z_{G}\right) / z_{A}}{G_{n}(x, z)}\right] \\
& +\Phi_{A} \frac{z}{z_{A}}
\end{aligned}
$$

where $\Phi_{O}=(1 / 2)\left(\Phi_{G}-\Phi_{A} z_{G} / z_{A}\right)\left[\ln \left(2 z_{G} / r_{o}\right)+M_{o}\right]^{-1}$, $F_{n}(x, z)=(x-n a)^{2}+\left(z+z_{G}\right)^{2}, G_{n}(x, z)=(x-n a)^{2}+$ $\left(z-z_{G}\right)^{2}, M_{o}=\sum_{l=1,2,3}^{\infty}, \ln \left[1+z_{G}^{2} /\left(\ell^{2} a^{2}\right)\right]$, and the sum

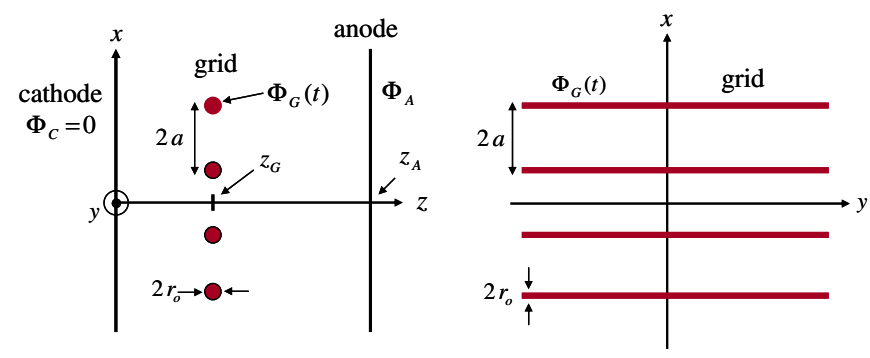

FIG. 4. Schematic of grid wires used for obtaining an estimate of the beam emittance due to the grid. The rings comprising the grid in Fig. 2 are modeled by an array of parallel grid wires. The grid wires, with radius $r_{o}$, are at a potential $\Phi_{G}$, are parallel to the $y$ axis, separated by a distance $2 a$, and located at $z=z_{G}$. 
is over the grid wires. The first term in the sum represents the potential due to the grid wires and their images from the cathode. The second term is a contribution induced by the electric field between the anode and cathode and is localized to the region around the wires. The last term in Eq. (1) represents the cathode-anode gap potential. The potential satisfies the appropriate boundary conditions on the cathode, grid, and anode surfaces.

The grid potential is the sum of a constant $(\mathrm{dc})$ and a time varying (rf) contribution, i.e., $\Phi_{G}(t)=\Phi_{\mathrm{DC}}+\Phi_{\mathrm{rf}}(t)$. The rf grid potential is given by $\Phi_{\mathrm{rf}}(t)=\Phi_{1} \cos \left(\omega_{\mathrm{rf}} t+\right.$ $\left.\varphi_{1}\right)+\Phi_{3} \cos \left(3 \omega_{\mathrm{rf}} t+\varphi_{3}\right)$, where $\omega_{\mathrm{rf}}$ is the fundamental linac frequency, $\Phi_{\ell}$ is the amplitude of the $\ell$ th harmonic, $\varphi_{\ell}$ is a constant phase, and $\ell=1$ or 3 . For parameters of interest the electrostatic approximation is valid, in the absence of beam loading effects, since the electron transit time $\tau_{\text {transit }} \sim z_{G} / \mathrm{v}_{z} \sim 30 \mathrm{psec}$ between the cathode and grid is small compared to the rf period, $\tau_{\mathrm{rf}}=2 \pi / \omega_{\mathrm{rf}} \sim$ $1.4 \mathrm{nsec}$.

Figure 5 illustrates the grid potential modulation that can be achieved using dc bias, fundamental, and 3rd harmonic. The figure schematically indicates the generation of short electron bunches of $\sim 100 \mathrm{psec}$ (rms) duration can be obtained at a repetition rate of $700 \mathrm{MHz}$. Shorter bunches can be achieved by the addition of higher harmonics, e.g., 5 th harmonic at $3.5 \mathrm{GHz}$.

Electron emission takes place when the axial electric field on the cathode surface is negative. The axial field on the cathode (in the absence of space-charge), from Eq. (C5) in Appendix C, is

$$
E_{z}(x, z=0)=-\Phi_{A} / z_{A}-\mu(x)\left(\Phi_{G} / z_{G}-\Phi_{A} / z_{A}\right),
$$

where $0<\mu(x)<1$ is a shielding parameter defined in Eq. (C6). The shielding parameter represents the reduction of the anode field due to the presence of the grid wires. The shielding of the electric field on the cathode is minimum

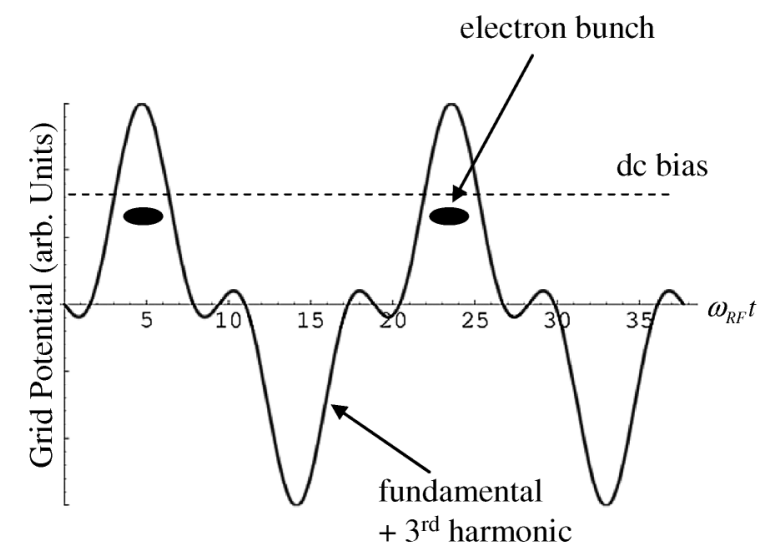

FIG. 5. Waveform consisting of the fundamental and third harmonic (blue curve). In this figure, the amplitude of the third harmonic is taken to be half that of the fundamental. The dc bias (dashed line) limits the electron bunch length to $\sim 90^{\circ}$ of the third harmonic, while filling every bucket.

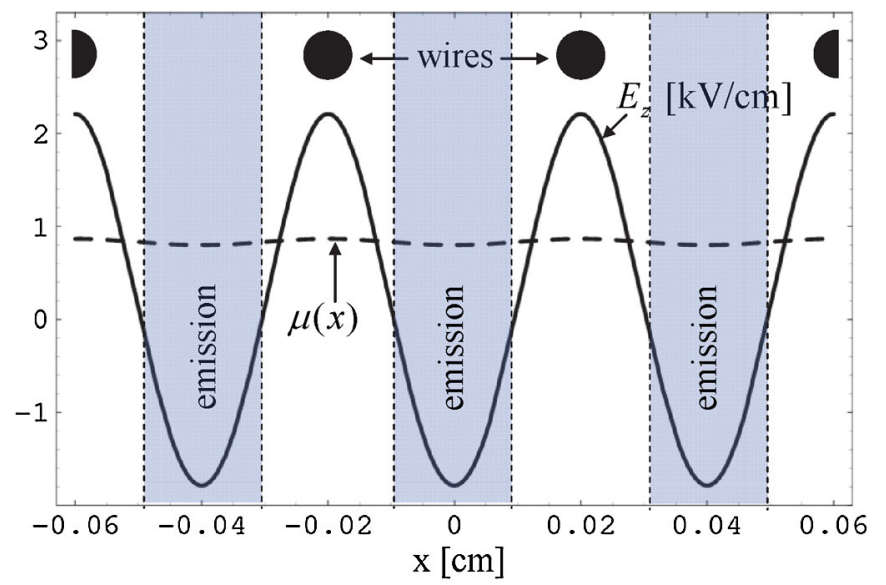

FIG. 6. Plot of shielding parameter $\mu(x)$ [Eq. (C6)] and the electric field on the cathode [Eq. (2)], versus transverse position for the configuration in Fig. 4. The grid is at a distance $z_{G}=$ $250 \mu \mathrm{m}$ from the cathode and the grid potential is $\Phi_{G}(t)=$ $-360-38 \cos \left(\omega_{\mathrm{rf}} t\right)+36 \cos \left(2 \omega_{\mathrm{rf}} t\right)-33 \cos \left(3 \omega_{\mathrm{rf}} t\right)$ in units of volts where the fundamental frequency is $f_{\text {rf }}=\omega_{\text {rf }} / 2 \pi=$ $700 \mathrm{MHz}$ and the plot is for $t=\pi / \omega_{\text {rf }}$. The cathode-anode gap is $z_{A}=1 \mathrm{~cm}$, the cathode-anode potential is $50 \mathrm{kV}$, and the grid wire radius is $r_{o}=30 \mu \mathrm{m}$. The shielding parameter, in the absence of space-charge, is $\mu=0.8$ between the wires and $\mu=0.87$ beneath the wires. It should be noted that the electron flux from the cathode is a sensitive function of $\mu(x)$. Electron emission from the cathode is transversely limited to the regions near the minima of $\mu(x)$.

between the wires and maximum beneath the wires. Typically for our parameters, $0.7<\mu(x)<0.9$. The shielding parameter controls electron emission and current density from the cathode. Figure 6 shows a plot of $\mu(x)$ and the axial electric field $E_{z}(x, z=0)$ on the cathode, extending over a region covering three grid wires for $t=\pi / \omega_{\mathrm{rf}}$. It should be noted that a small variation in $\mu(x)$ is sufficient to cause a relatively large change in $E_{z}$ and electron flux across the surface.

\section{Transverse bunch emittance}

There are several sources that contribute to the emittance of the electron bunch $[26,27,33]$. For a cathode radius of $R_{c}=1 \mathrm{~cm}$, the contribution to the normalized emittance from the finite cathode temperature is $\varepsilon_{n, T}=$ $(1 / 2) R_{c}\left(k_{B} T_{c} / m c^{2}\right)^{1 / 2} \approx 2 \mathrm{~mm} \mathrm{mrad}$, where $T_{c}=$ $1300 \mathrm{~K}$ is the cathode temperature. The emittance contribution due to a magnetic field on the cathode is $\varepsilon_{n, B}=$ $q B_{o} R_{c}^{2} / 2 m c^{2} \approx 1.4 \mathrm{~mm}$ mrad, where $B_{o}=0.5 \mathrm{G}$ is the assumed magnetic field on the cathode from the Earth and other stray sources. Roughness on the cathode contributes to the emittance by an amount on the order of $\varepsilon_{n, R}=$ $\pi h /(2 \ell) R_{c}\left(q E_{S} h / 2 m c^{2}\right)^{1 / 2} \sim 0.5 \mathrm{mmmrad}$, where $h=$ $1 \mu \mathrm{m}$ is the amplitude of surface roughness, $\ell=20 \mu \mathrm{m}$ is the period of the roughness, $E_{S}=5 \mathrm{kV} / \mathrm{cm}$ is the surface field. 


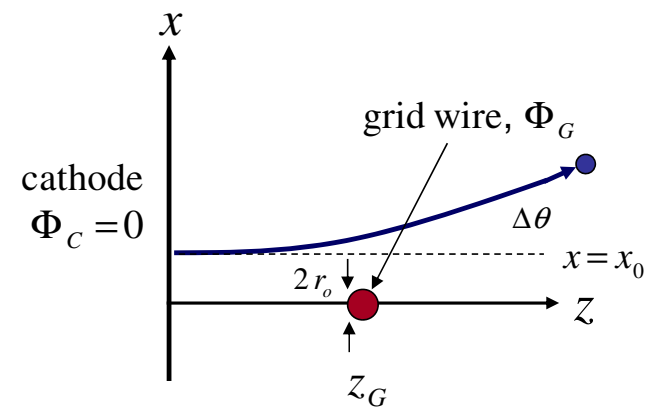

FIG. 7. Calculation of emittance due to a single grid wire. Here the cathode is at ground potential and located at $z=0$, the grid wire is at potential $\Phi_{G}$, located at $z=z_{G}$, and has radius $r_{o}$. An electron with impact parameter $x_{0}$ is deflected through an angle $\Delta \theta$ due to grid wire.

The emittance due to a single grid wire, as shown in Fig. 7, in the absence of self-fields, is given by (Appendix C)

$$
\varepsilon_{n, \text { grid }} \approx R_{b}\left\langle\beta_{x}\right\rangle \approx \frac{2 \mu_{1}(a)}{3}\left(\frac{a}{z_{G}}\right) R_{b} \frac{\left|\bar{\Phi}_{G}-\left(z_{G} / z_{A}\right) \bar{\Phi}_{A}\right|}{\left(z_{G} / z_{A}\right)^{1 / 2} \bar{\Phi}_{A}^{1 / 2}},
$$

where $R_{b} \approx R_{c}$ is the beam radius, $\left\langle\beta_{x}\right\rangle$ is the averaged normalized electron velocity, $\mu_{1}(a)$ is the shielding parameter due to one wire (Appendix C), $\bar{\Phi}_{G}=$ $|q| \Phi_{G} /\left(m c^{2}\right)$, and $\bar{\Phi}_{A}=|q| \Phi_{A} /\left(m c^{2}\right)$. Using typical values for the gun parameters, the normalized emittance due to the grid is $\varepsilon_{n \text {,grid }}[\mathrm{mm} \mathrm{mrad}] \sim 5-10 R_{b}[\mathrm{~cm}]$. This range of emittances is consistent with PIC simulation results that are presented in the next section.

The nonrandom, macroscopic contributions to the beam emittance, e.g., due to focusing/accelerating fields and space-charge fields, can be reduced through the use of emittance compensating coils or optimizing the anode geometry. The microscopic and random contributions to emittance, e.g., cathode temperature, cathode surface roughness, grid fields, or slice emittance, cannot be reduced using emittance compensating coils. From PARMELA simulations, it is known that the dominant contribution to the transverse emittance is the nonlaminar flow induced by the cathode/anode geometry, and the grid fields. Additional work is under way to understand the various contributions to the emittance and to reduce emittance growth due to space-charge and macroscopic nonlinear effects by changing the gun geometry and the use of emittance compensation coils [34].

\section{PIC SIMULATIONS}

Accurate numerical simulation of the injector requires the modeling of complicated geometries and numerous physical processes, not all of which are contained in any single code. For example, TURBOWAVE [21] can model fully electromagnetic particle fields and image charges
TABLE II. Parameters used for PARMELA simulation of an rfgated, gridded thermionic gun for a high-average-power FEL. The gun geometry is shown in Fig. 19. Bunch diagnostics are provided at the gun exit at $z=5.0$.

\begin{tabular}{ll}
\hline \hline Cathode-grid gap, $z_{G}$ & $250 \mu \mathrm{m}$ \\
Cathode-anode gap, $z_{A}$ & $1.4 \mathrm{~cm}$ \\
Grid wire radius, $r_{o}$ & $30 \mu \mathrm{m}$ \\
Grid wire separation, $2 a$ & $450 \mu \mathrm{m}$ \\
Cathode radius, $R_{c}$ & $1.5 \mathrm{~cm}(0.9 \mathrm{~cm}$ active) \\
Peak cathode field, $\left|E_{z}\right|_{\max }$ & $6.5 \mathrm{kV} / \mathrm{cm}$ \\
Cathode-anode voltage, $\Phi_{A}$ & $35 \mathrm{kV}$ \\
rf grid modulation frequency, $f_{\mathrm{rf}}$ & $700 \mathrm{MHz}, 2.1 \mathrm{GHz}$ \\
Bunch charge, $q N_{b}$ & $1 \mathrm{nC}$ \\
Average energy, $E$ & $35 \mathrm{kV}$ \\
Energy spread (rms) & $1 \%$ \\
Pulse length (rms), $\tau_{b}$ & $45 \mathrm{psec}$ \\
Transverse emittance (rms), $\varepsilon_{n, x}$ & $16 \mathrm{~mm} \mathrm{mrad}$ \\
Longitudinal emittance (rms), $\varepsilon_{z}$ & $7 \mathrm{keV} \mathrm{psec}$ \\
\hline \hline
\end{tabular}

induced on the cathode, anode, and grid wires, but is difficult to use with the complicated geometry of a realistic cathode-grid design. PARMELA [22] on the other hand, can handle more complicated geometries, but does not calculate image fields correctly, i.e., the image fields due to the grid and anode are neglected. In this section, we present a number of simulations using both TURBOWAVE and PARMELA to illustrate the basic operation of a thermionic injector for a high-power FEL. The injector geometry is based on an inductive output tube (IOT) gun design [35] and the basic parameters used in the PARMELA simulations are listed in Table II. Because of the different geometries used in the TURBOWAVE (2D axisymmetric, planar cathode and grid) and PARMELA simulations (2D axisymmetric, curved cathode and grid), the parameters used differ between the two codes. The PARMELA injector simulation that uses a filled cathode (see Fig. 19) produces electron bunches with characteristics required for high-power FEL operation, as specified in Fig. 1. However, this simulation should serve only as a basic illustration of high-power injector operation and not an actual injector design. An injector design would require further optimization of the cathode, grid, and anode geometry.

\section{A. TURBOWAVE}

TURBOWAVE is a $2 \mathrm{D}$ or $3 \mathrm{D}$, fully relativistic PIC code that models a variety of physical processes. Here we describe two-dimensional slab and axially symmetric electrostatic simulations of the gun. In these simulations the cathode and anode are held at fixed potentials, while the grid consists of parallel wires or concentric rings whose potential is a prescribed function of time. The potential at all other locations within the gun region is computed via successive over-relaxation, and includes space-charge effects. 
Figure 8 shows an illustration of the electron dynamics including space-charge effects in the cathode-grid region. In this simulation an infinite array of parallel wires using a 2D slab geometry was employed. The grid potential is $\Phi_{G}(t)=-360-38 \cos \left(\omega_{\mathrm{rf}} t\right)+36 \cos \left(2 \omega_{\mathrm{rf}} t\right)-$ $33 \cos \left(3 \omega_{\mathrm{rf}} t\right)$ in units of volts and the fundamental frequency is $f_{\mathrm{rf}}=\omega_{\mathrm{rf}} / 2 \pi=700 \mathrm{MHz}$. The cathodeanode gap is $z_{A}=1 \mathrm{~cm}$ and the cathode-anode potential is $50 \mathrm{kV}$. The grid wire radius is $r_{o}=30 \mu \mathrm{m}$. A constant electron density is maintained on the cathode and the electrons are emitted and propagated by the external and space-charge fields. The time-integrated current density is shown in Fig. 8 along with potential contours at the peak of emission. Only three spatial periods of the infinite grid structure are shown in the figure. The particles are primarily emitted between the wires so that the grid current (grid heating) is very low. This is due to the shielding effect of the grid wires on the electric field, as discussed in Appendix C. The shielding parameter for the parameters of Fig. 8 is $\mu=0.8$ between the wires and $\mu=0.87$ beneath the wires.

As another example, TURBOWAVE is used in cylindrical geometry to simulate the ring grid configuration shown in Fig. 9. The configuration of the gun shown in Fig. 9 includes focusing electrodes, at 45 degrees, which are found to reduce the emittance. The current pulses produced at the grid plane and the anode plane are shown in Fig. 10, along with the time varying potential of the grid wires. In this example, $60 \mathrm{psec}(\mathrm{rms})$ bunches having a charge of $0.7 \mathrm{nC}$ are generated. The average current is $\left\langle I_{b}\right\rangle=0.5 \mathrm{~A}$. Figure 11 shows the slice emittance, which at the peak of the current pulse is $\sim 5 \mathrm{~mm}$ mrad. The projected transverse phase space, shown in Fig. 12, corresponds to a projected emittance of $\varepsilon_{n, \perp}=8.9 \mathrm{~mm}$ mrad. This is also shown as the horizontal dashed line in Fig. 11. The longitudinal

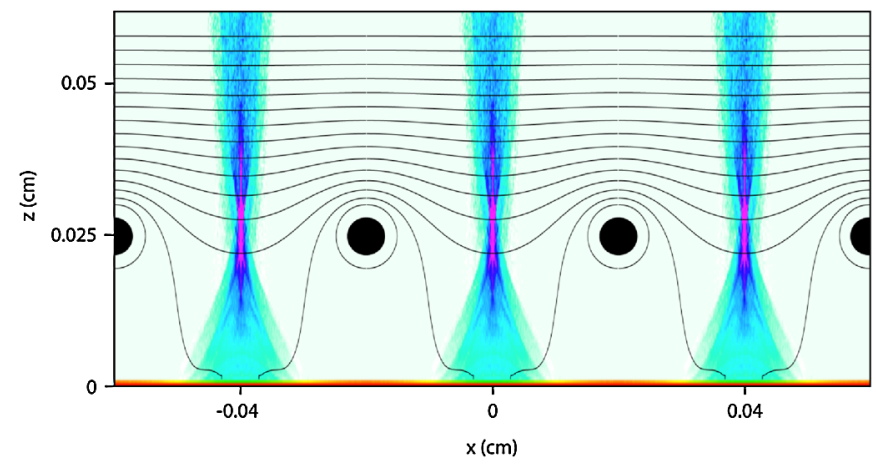

FIG. 8. TURBOWAVE simulations of the cathode-grid region including the effects of space-charge. The simulation geometry is 2D Cartesian slab. The color scale indicates time-averaged current density, with red more dense and blue less dense. The cross sections of four grid wires are shown. The peak current density between the wires is $150 \mathrm{~A} / \mathrm{cm}^{2}$. The total charge associated with a single beamlet is $45 \mathrm{pC} / \mathrm{cm}$. The cathode is located at $z=0$, the lower boundary of the figure. Parameters are the same as in Fig. 6. Black curves show the equipotentials.

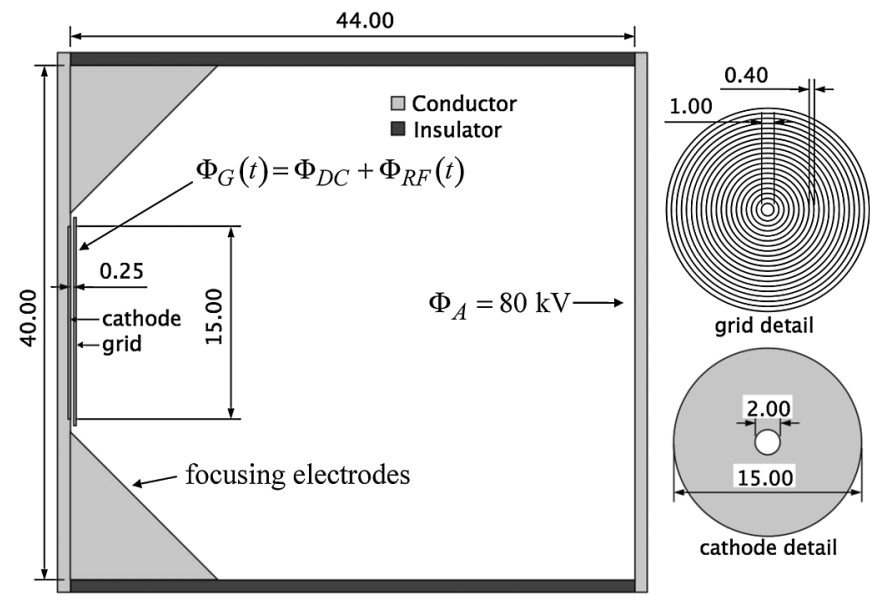

FIG. 9. Gun geometry for TURBOWAVE PIC simulations. The closed box on the left is bounded by insulators on the top and the bottom, the cathode on the left, and anode on the right. The $45^{\circ}$ focusing electrodes are also shown. The cathode is grounded, the anode is at $80 \mathrm{kV}$ and the grid potential consists of a dc contribution $\Phi_{\mathrm{DC}}$, and a time-varying rf contribution $\Phi_{\mathrm{rf}}(t)$. The cross section of the cathode and the grid are shown on the right. All dimensions are in $\mathrm{mm}$.

phase space shown in Fig. 13 is characterized by a longitudinal emittance of $\varepsilon_{z}=20 \mathrm{keV}$ psec.

TURBOWAVE has also been used to determine the effect of beam loading in the cathode-grid region. A simple estimate indicates that beam loading is not important for

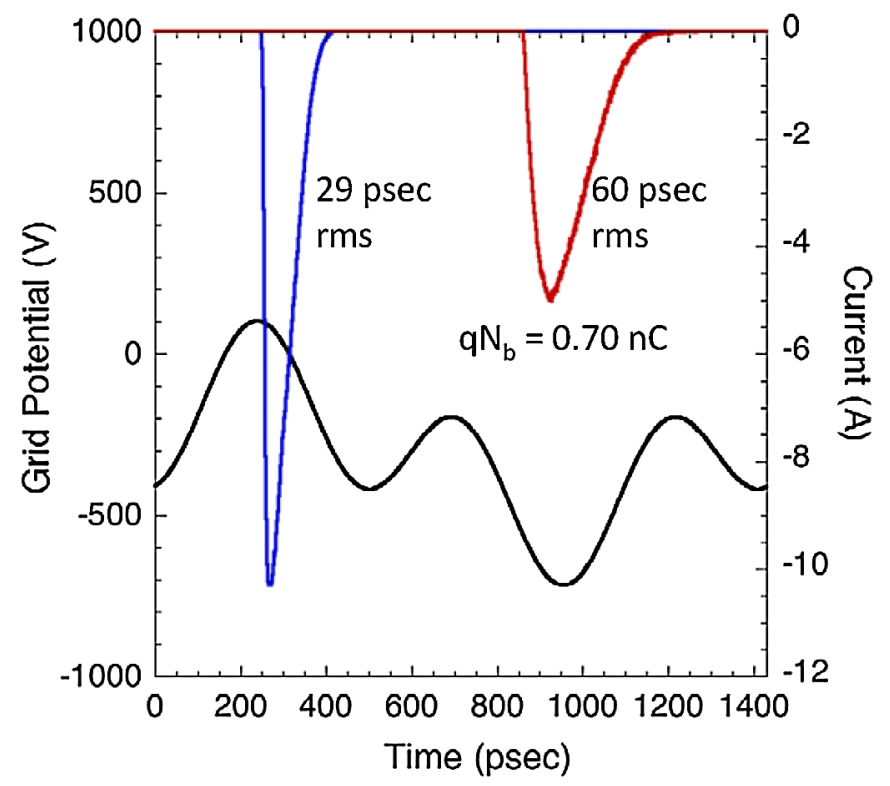

FIG. 10. TURBOWAVE simulations of current pulses at the grid and at the anode for the geometry shown in Fig. 9. The current pulses produced at the grid plane and the anode plane are shown in blue and red, respectively, while the time varying potential on the grid wires consisting of a dc and $\mathrm{rf}$ contributions is shown in black. A bunch with a $60 \mathrm{psec}$ (rms) duration and $0.7 \mathrm{nC}$ of charge crosses the anode resulting in an average current of $\left\langle I_{b}\right\rangle=0.5$ A. 


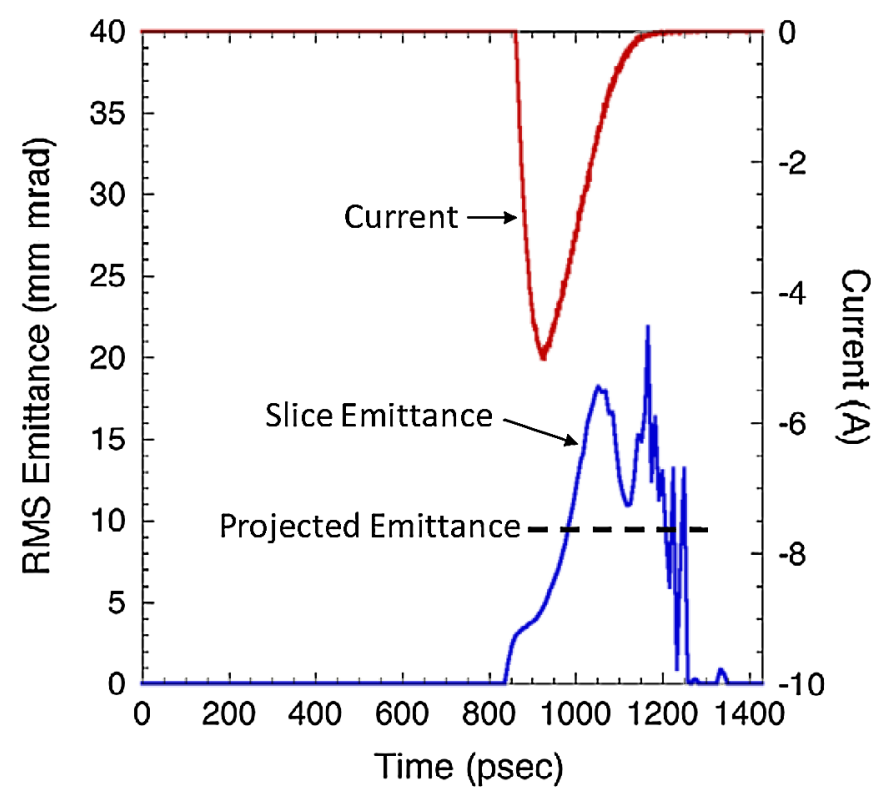

FIG. 11. Simulated current and emittance at the anode for the geometry shown in Fig. 9. The red curve shows the current and the blue curve shows the slice emittance. The slice emittance at the peak of the current pulse is $\varepsilon_{n, \perp} \sim 5 \mathrm{~mm}$ mrad. The projected emittance $\varepsilon_{n, \perp}=8.9 \mathrm{~mm} \mathrm{mrad}$ is shown by the dashed horizontal line.

the parameters under consideration. The rf fill time, i.e., the time it takes to fill the cathode-grid region with rf energy, is $\tau_{F} \approx R_{c} / c \sim 30 \mathrm{psec}$, which is short compared to the $\mathrm{rf}$ period, $\tau_{\mathrm{rf}} \approx 1.4 \mathrm{nsec}$. In addition, for the parameters being considered the ratio of the kinetic energy of the electron bunch crossing the grid plane to the rf energy in the cathode-grid region is $W_{b} / W_{\mathrm{rf}}<1$, where $W_{b} \sim$ $q N_{b} \Phi_{G}, \quad W_{\mathrm{rf}}=(2 / 15)\left[R_{c}^{2} /\left(z_{G} r_{e}\right)\right]\left(q \Phi_{G} / m c^{2}\right) q \Phi_{G}$, and

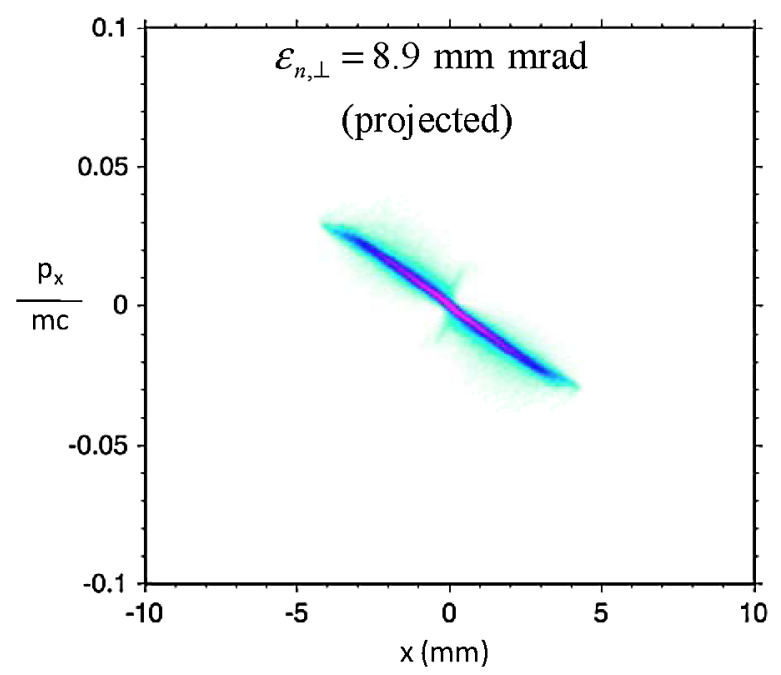

FIG. 12. Projected transverse phase space for the gun geometry of Fig. 9. The ordinate is the $x$ component of the momentum normalized to $m c$ and the abscissa is the $x$ coordinate. The projected normalized emittance is $\varepsilon_{n, \perp}=8.9 \mathrm{~mm} \mathrm{mrad}$.

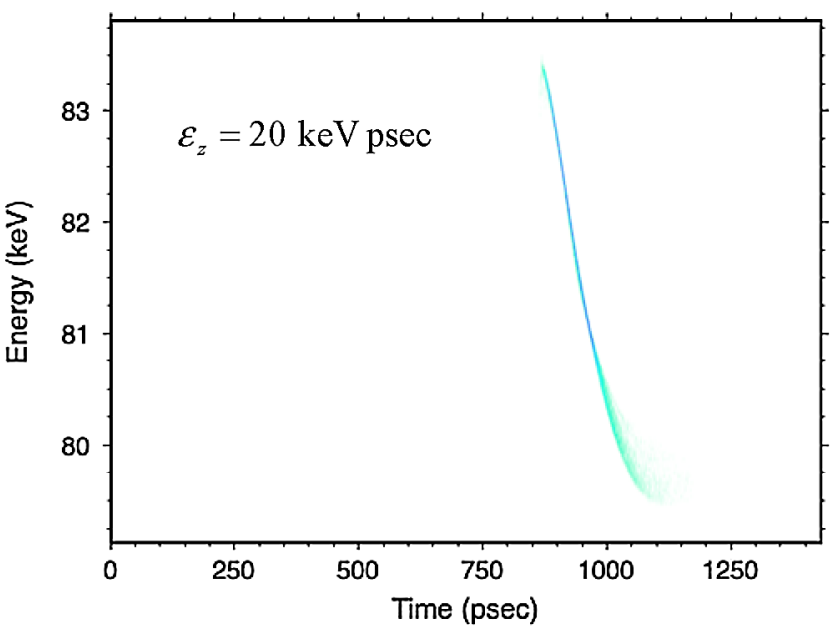

FIG. 13. Longitudinal phase space (energy vs time) for the gun geometry of Fig. 9. The normalized longitudinal emittance is $\varepsilon_{z}=20 \mathrm{keV}$ psec.

$r_{e}=q^{2} / m c^{2}$. Since $W_{b} / W_{\mathrm{rf}}<1$ and the electron bunch duration at the grid plane $\tau_{b} \sim 100 \mathrm{psec}$ is greater than $\tau_{F}$, the rf energy in the cathode-grid region is not significantly depleted by the electron bunch. Simulations using

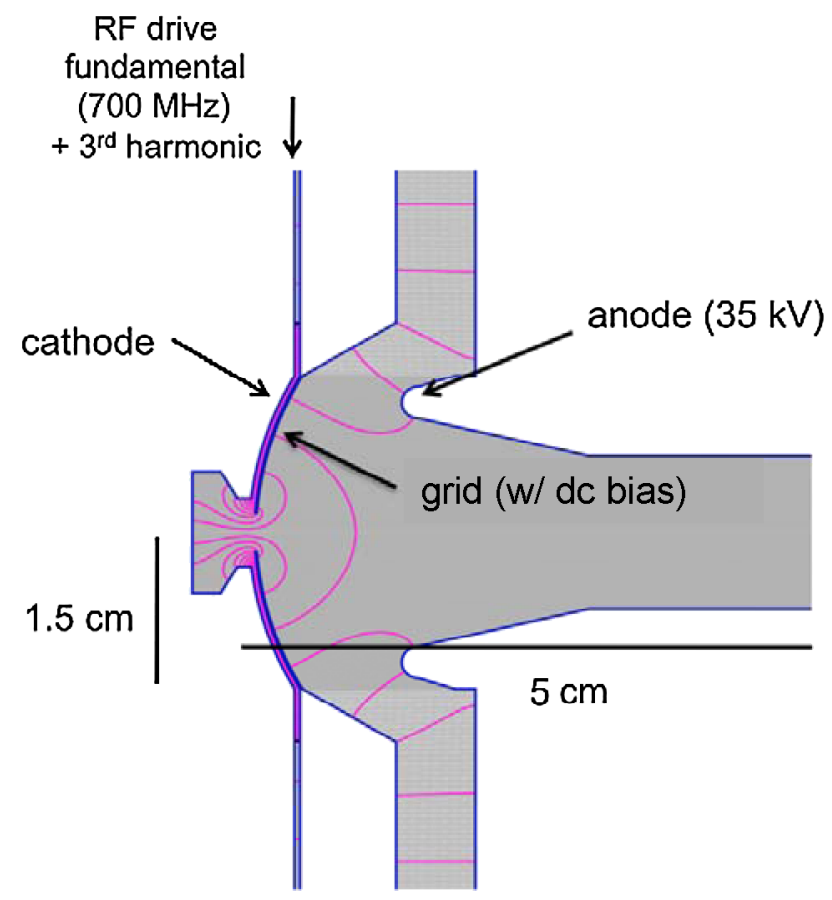

FIG. 14. Geometry of a thermionic IOT gun used for the PARMELA simulations of Fig. 17. The cathode-grid gap is $0.25 \mathrm{~mm}$. Fields driven at the fundamental $(700 \mathrm{MHz})$ and third harmonic are injected into the cathode-grid region, and the grid has a bias of $-450 \mathrm{~V}$. The rf fields were computed using SUPERFISH, while the dc fields were computed using POISSON. Field lines of the fundamental $(700 \mathrm{MHz})$ field are shown. The time-dependent potential at the location of the grid peaks at $160 \mathrm{~V}$. The three-dimensional geometry is symmetric about an axis along the center of the figure. 

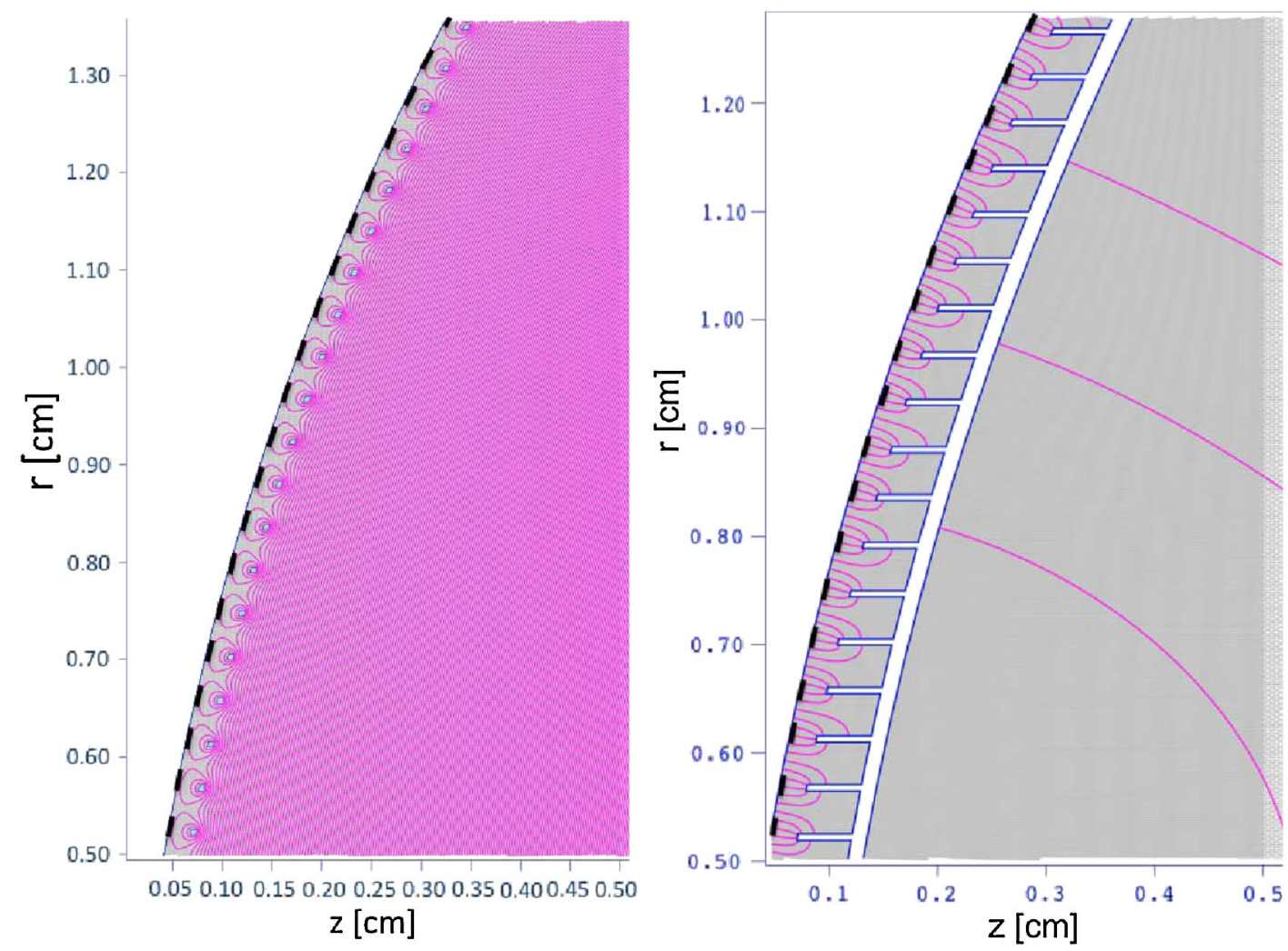

FIG. 15. Close-up of the grid region used in the PARMELA simulations of Fig. 17. Black lines illustrate the shadow grid used to prevent emission from the cathode beneath the grid wires. (Left) For the purpose of computing the static field, the grid is modeled as a series of concentric rings of radius $r_{o}=30 \mu \mathrm{m}$. Curves denote contours of the electric potential due to the bias voltages on the anode and the grid. (Right) For the purpose of computing the rf field, the grid is modeled as a series of thin, concentric rings attached to a curved backing plate which confines the rf field to the grid-cathode region. The backing plate is transparent to electrons in the simulations. Curves denote field lines of the fundamental $(700 \mathrm{MHz})$ rf field.

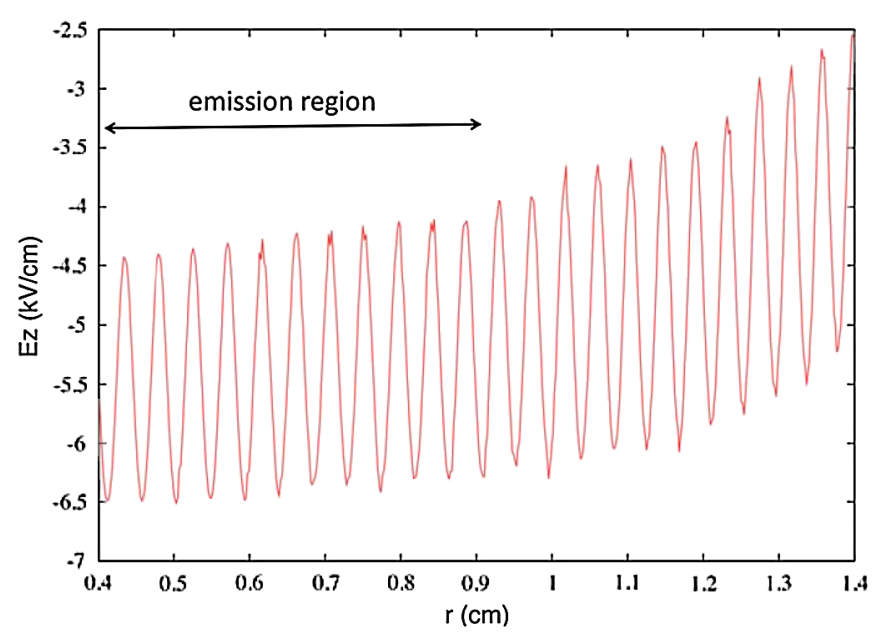

FIG. 16. Electric field at the cathode used for PARMELA simulations. The total electric field across the cathode surface is plotted versus radial distance from the symmetry axis. At a given point on the cathode, the field takes the form $E_{z}=E_{z, \mathrm{DC}}+$ $E_{z, 1} \sin \left(\omega_{\mathrm{rf}} t\right)+E_{z, 3} \sin \left(3 \omega_{\mathrm{rf}} t\right), \quad$ where $\quad E_{z, \mathrm{DC}} \approx 11 \mathrm{kV} / \mathrm{cm}$, $E_{z, 1} \approx-9.4 \mathrm{kV} / \mathrm{cm}$, and $E_{z, 3} \approx 9 \mathrm{kV} / \mathrm{cm}$. The field is illustrated for $t=\pi / 2 \omega_{\text {rf }}$ at the peak of emission. Emission occurs only at those radii within the indicated region.
TURBOWAVE are consistent with the above arguments that beam loading for the parameters under consideration is not significant.

\section{B. PARMELA}

IOT guns have a number of characteristics necessary for use as injectors for high-power FELs, e.g., high average currents, short bunch lengths, long lifetimes, robustness, etc. However, because the applications for which IOT guns were designed did not require low emittance, the transverse bunch emittance is typically too large for use as a high average power FEL injector. In this subsection, we use the PARMELA code to simulate the complicated geometry and field structures of two typical IOT gun designs and characterize the emitted electron bunches. We show that, through the use of fundamental and third harmonic rf frequencies, the bunch length is sufficiently short and the average current sufficiently high for use as a high-power FEL injector. While the resulting emittance is large (15-25 mm mrad), it should be possible to modify the present gun design to lower the emittance, for example, by improving the anode and cathode geometry. 

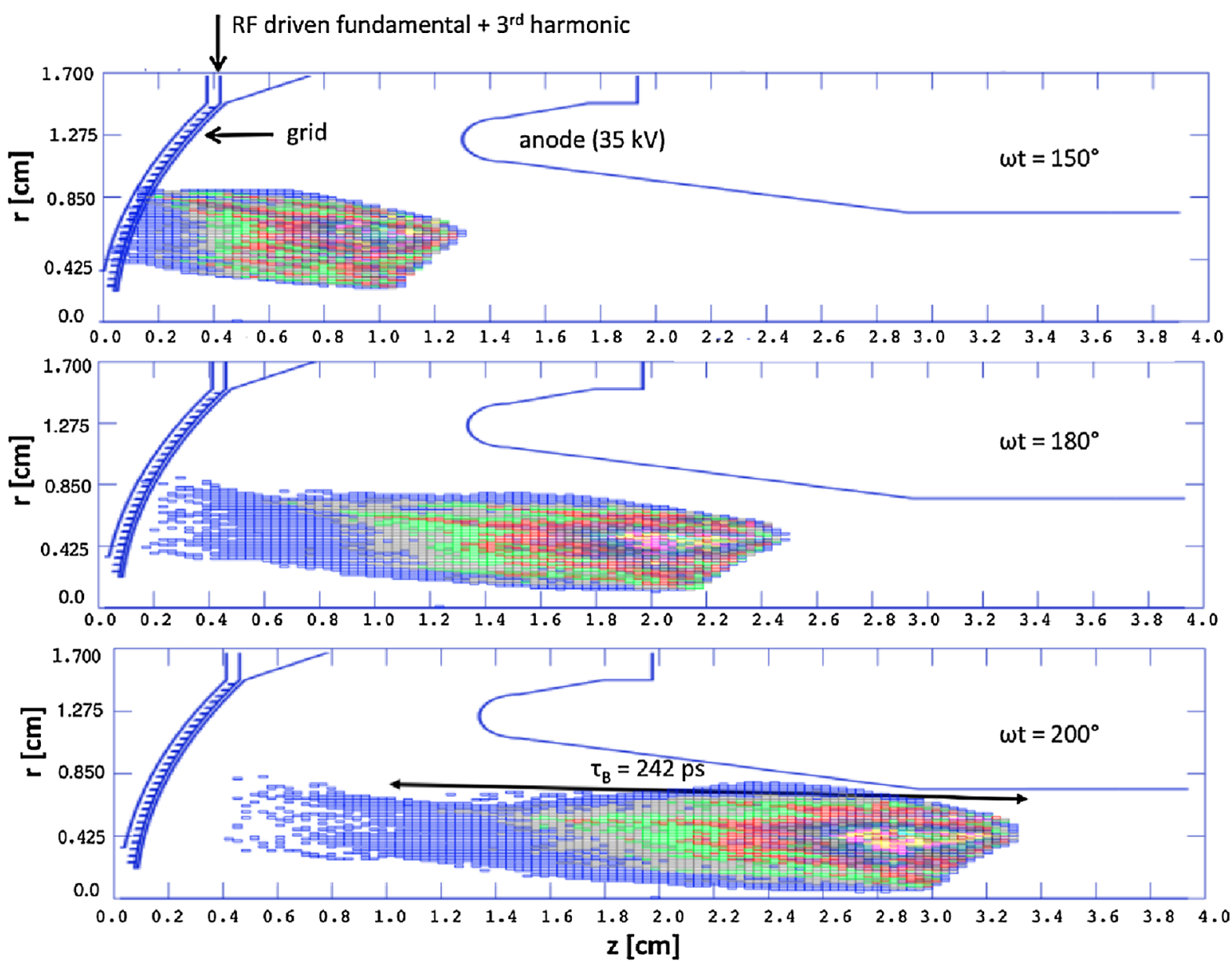

FIG. 17. PARMELA simulation of an rf-gated, gridded thermionic gun. The plots show the generation of a $1 \mathrm{nC}$ electron bunch with end-to-end duration of $242 \mathrm{psec}$ and a normalized emittance of $25 \mathrm{~mm}$ mrad. The grid is biased to $-450 \mathrm{~V}$ dc, and the anode is biased to $35 \mathrm{kV}$ relative to the cathode. The amplitude of the rf field in the cathode-grid gap is $9.4 \mathrm{kV} / \mathrm{cm}$. The rf phase $\omega t$ is indicated in each figure.
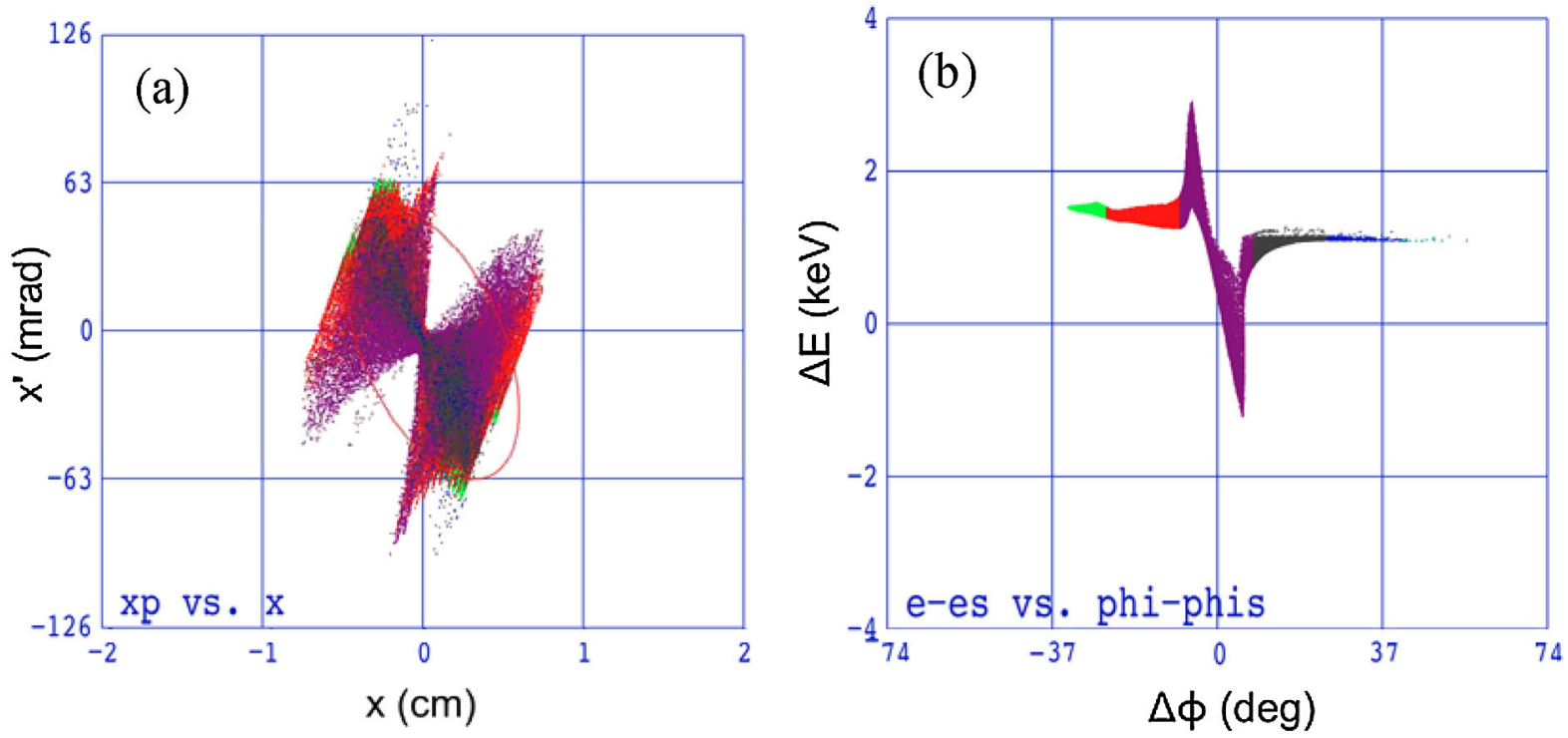

FIG. 18. (a) Transverse and (b) longitudinal phase space from the PARMELA simulation of Fig. 17 at the exit of the gun $(z=5.0 \mathrm{~cm})$. The normalized transverse emittance is $25 \mathrm{~mm}$ mrad and the longitudinal emittance is $17 \mathrm{keV}$ psec. Particles are grouped by color into six longitudinal slices, with each slice containing particles of a given range in rf phase at $z=5.0 \mathrm{~cm}$. 


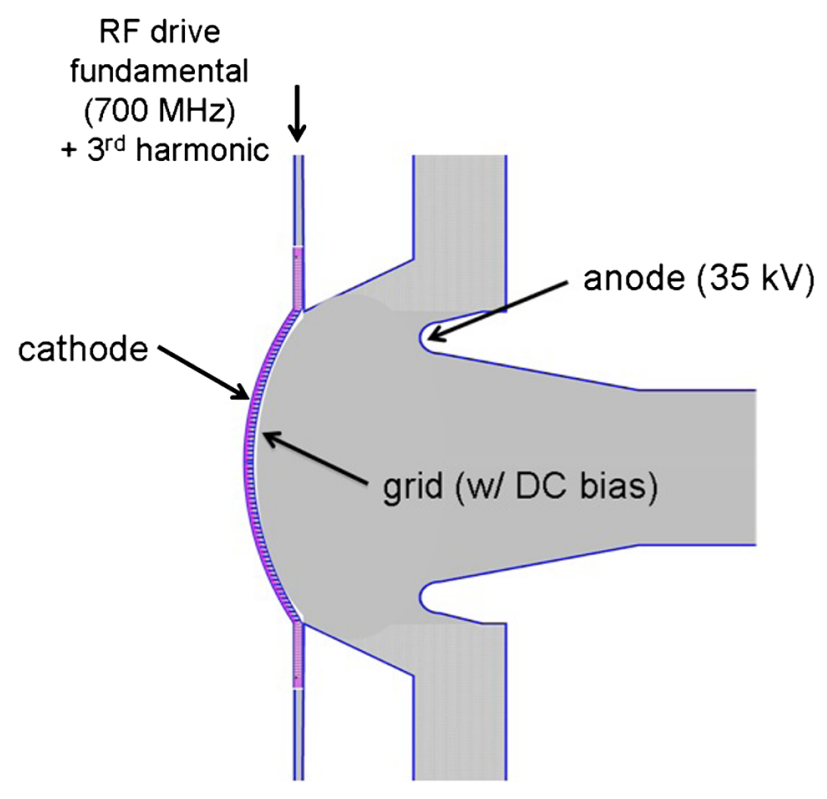

FIG. 19. Geometry of a thermionic IOT gun with a closed cathode (compare Fig. 14), used for the PARMELA simulations of Fig. 20. Field lines of the fundamental (700 MHz) field are shown. In these simulations, the backing plate entirely confines the rf fields to the grid-cathode region. The three-dimensional geometry is symmetric about an axis along the center of the figure.

The first geometry used for PARMELA [22] simulations is shown in Figs. 14 and 15. To preserve the azimuthal symmetry of the simulation, the grid (Fig. 15) is modeled as a series of thin, concentric rings separated by a distance of $0.4 \mathrm{~mm}$. For the purpose of computing the rf field, this grid is attached to a curved backing plate that confines the rf field within the grid-cathode region. In an actual gun, this backing plate is replaced by a number of radial spokes that serve to contain the rf field. In the simulations, the backing plate is transparent to electrons. A shadow grid is used to prevent electron emission directly under the wires. For the present parameters the use of a shadow grid reduces the emittance by $\sim 10 \%$.

The grid is biased to $-450 \mathrm{~V}$ dc relative to the cathode, and an $\mathrm{rf}$ field at the frequency of the fundamental $(700 \mathrm{MHz})$ and the third harmonic is injected radially into the cathode-grid gap. The fundamental rf field has an amplitude of $9.4 \mathrm{kV} / \mathrm{cm}$, while the anode is maintained at a potential of $35 \mathrm{kV}$. The temporal profile of the total $\mathrm{rf}$ field is illustrated schematically in Fig. 5. The total longitudinal field at the cathode surface is illustrated in Fig. 16.

To simulate emission at the cathode, a long, low-velocity $(0.1 \mathrm{eV})$ bunch is initialized behind the cathode surface to serve as a reservoir of charge. The bunch is spatially uniform over the cross section $0.4 \mathrm{~cm}<r<0.9 \mathrm{~cm}$, and no particles are contained in those radii corresponding to the locations of the shadow grid wires. As a particle in this bunch crosses the cathode surface, it is emitted only if it lies within the range of rf phase indicated in Fig. 5, and emission occurs over the cathode region indicated in Fig. 16. Particles that are turned around at the cathode surface are absorbed, and do not contribute to the computation. The emitted electrons are propagated in the external and space-charge fields, and image charges at the cathode surface are included.

Figure 17 is a series of plots showing the evolution of the electron bunch as it starts from the cathode and travels a distance of $5 \mathrm{~cm}$ to the end of the gun. In this example, $46 \mathrm{psec}$ (rms) bunches having a charge of $1 \mathrm{nC}$ are generated. The average current is $\left\langle I_{b}\right\rangle=0.7 \mathrm{~A}$. The transverse phase space at the exit of the gun, as shown in Fig. 18(a), corresponds to a projected emittance of $\varepsilon_{n, \perp}=$ $25 \mathrm{~mm}$ mrad, while the slice emittance is $\sim 23 \mathrm{~mm} \mathrm{mrad}$. The longitudinal phase space is shown in Fig. 18(b), and is characterized by a longitudinal emittance of $\varepsilon_{z}=$ $17 \mathrm{keV}$ psec.

The transverse emittance can be reduced by modifying the cathode-anode geometry. As an example, the hole in the cathode was closed to produce the gun geometry illustrated in Fig. 19. The grid is again modeled as illustrated in Fig. 15 using the parameters shown in Table II. The field profile at the cathode is the same as that illustrated in Fig. 16, except that the emission region extends to $r=0$. Figure 20 is a series of plots showing the evolution of the electron bunch as it starts from the cathode and travels a distance of $5 \mathrm{~cm}$ to the end of the gun. In this example, $45 \mathrm{psec}$ (rms) bunches having a charge of $1.0 \mathrm{nC}$ are generated. The average current is $\left\langle I_{b}\right\rangle=0.7 \mathrm{~A}$. The transverse phase space at the exit of the gun, as shown in Fig. 21(a), corresponds to a projected emittance of $\varepsilon_{x, n}=$ $16 \mathrm{~mm} \mathrm{mrad}$, while the slice emittance is $\sim 14 \mathrm{~mm} \mathrm{mrad}$. The longitudinal phase space is shown in Fig. 21(b), and is characterized by a longitudinal emittance of $\varepsilon_{z}=$ $7 \mathrm{keV}$ psec. Note that the transverse emittance has been reduced by $40 \%$, while the pulse length and average current are essentially unchanged.

These simulations illustrate that it is possible to obtain from a conventional IOT gun the high average currents and short bunch lengths necessary for use as an injector for a high-power FEL. Through improved cathode-anode geometry, it is possible that a similar design may produce smaller transverse emittances with $\varepsilon_{x, n}<15 \mathrm{~mm}$ mrad.

\section{CONCLUSION}

In this paper we have proposed, analyzed, and simulated an rf-gated, gridded thermionic gun for high average power IR FELs. Simulations using TURBOWAVE and PARMELA indicate that the necessary electron beam parameters for the FEL, i.e., charge per bunch, average current, quality, and pulse format, can be achieved. The beam emittance induced by the grid is within acceptable limits for operation in the IR regime. Additional work is under way to understand the various contributions to the emittance and to reduce emittance growth due to space-charge and 

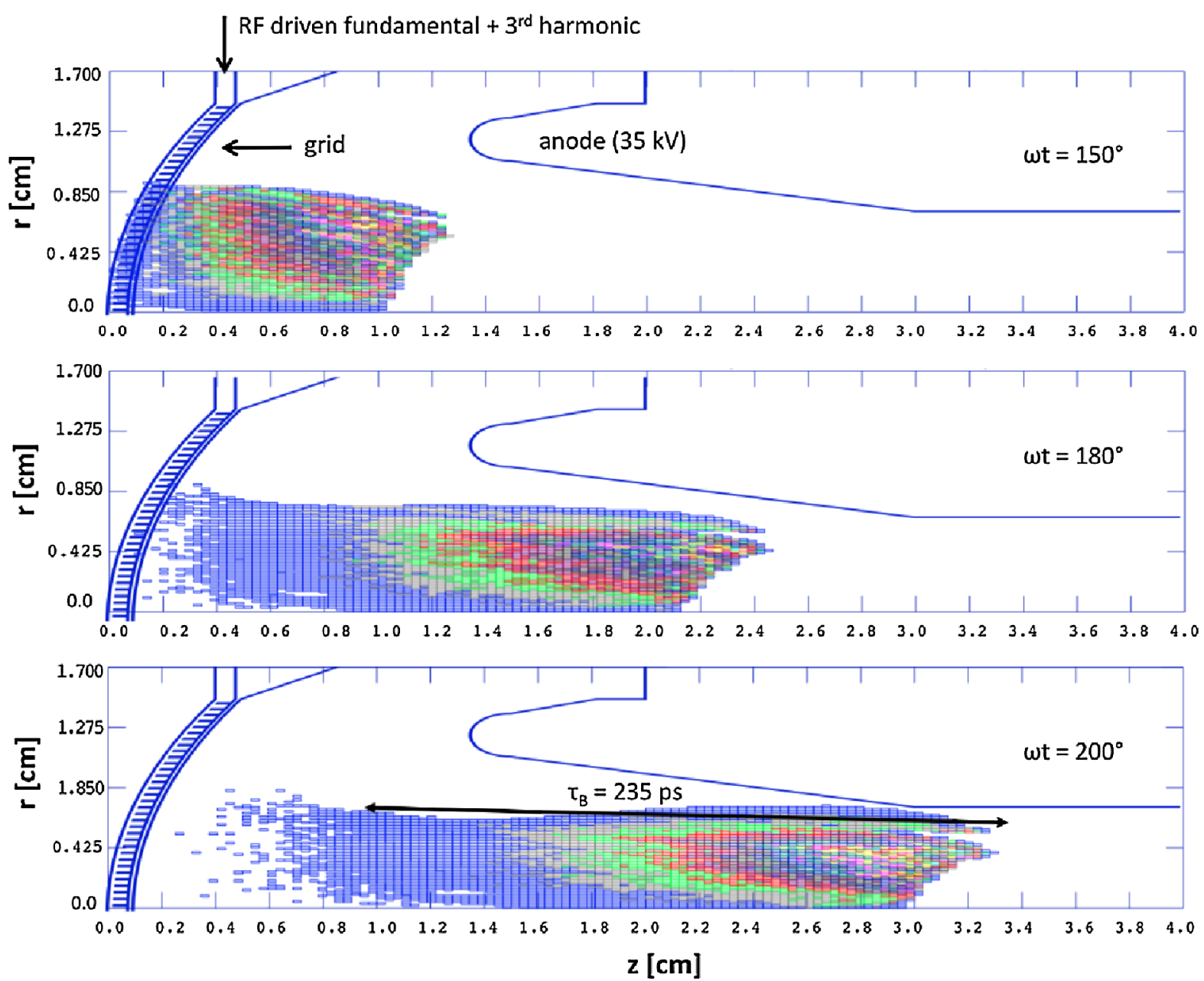

FIG. 20. PARMELA simulation of the rf-gated, gridded thermionic gun illustrated in Fig. 19 (compare Fig. 17). The plots show the generation of a $1 \mathrm{nC}$ electron bunch with end-to-end duration of $235 \mathrm{psec}$ and a normalized emittance of $16 \mathrm{~mm}$ mrad. The rf phase $\omega t$ is indicated in each figure.
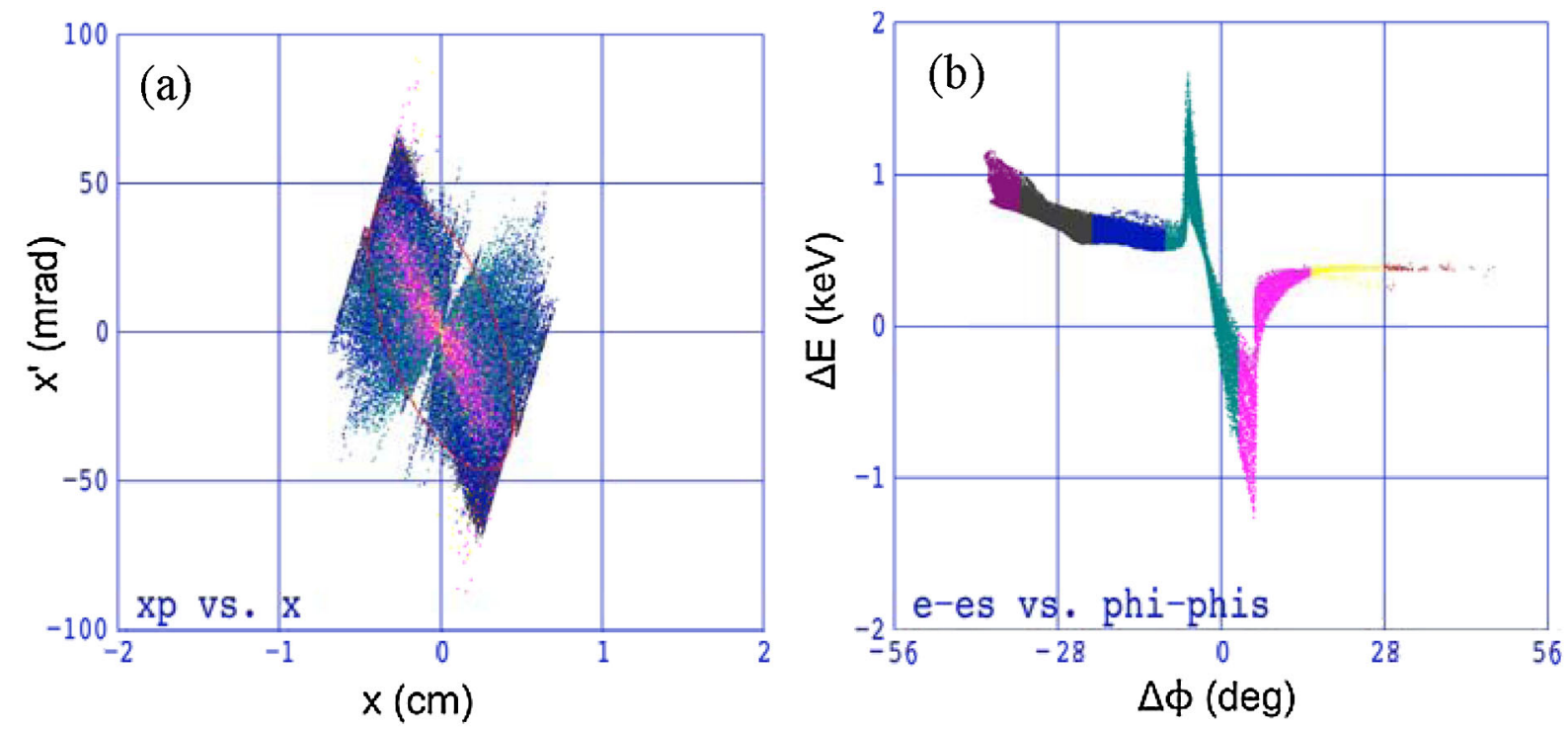

FIG. 21. (a) Transverse and (b) longitudinal phase space from the PARMELA simulation of Fig. 20 at the exit of the gun $(z=5.0 \mathrm{~cm})$. The normalized transverse emittance is $16 \mathrm{~mm}$ mrad and the longitudinal emittance is $7 \mathrm{keV}$ psec. Particles are grouped by color into six longitudinal slices, with each slice containing particles of a given range in rf phase at $z=5.0 \mathrm{~cm}$. 
macroscopic nonlinear effects by changing the gun geometry and the use of emittance compensation coils [34].

The required high average current can be obtained with dispenser or single-crystal thermionic cathodes, which are robust and have long lifetimes. Thermionic cathodes can provide the necessary high average current, charge per bunch, and pulse format for high average power FELs. They operate at moderate vacuums $\left(10^{-8}-10^{-7}\right.$ Torr $)$, have long lifetimes ( $>10000$ hours) delivering over $10^{8}$ coulombs and can provide cathode current densities $>10 \mathrm{~A} / \mathrm{cm}^{2}$. The pulse duration requirements can be met by modulating the grid with a combination of the fundamental and 3rd harmonic of the linac frequency, filling every rf bucket in the linac. The heat radiated by the thermionic gun in the proposed designed is relatively small and, hence, it can be configured with either normalconducting or superconducting prebooster cavities. An experimental program is under way at the Naval Research Laboratory to demonstrate this high average current electron gun configuration.

\section{ACKNOWLEDGMENTS}

The authors are grateful to Professor P. Serafim for useful discussions. This work was supported by the Office of Naval Research. C. Mitchell is supported by a National Research Council postdoctoral fellowship.

\section{APPENDIX A: ELECTRON BEAM QUALITY REQUIREMENTS FOR FELS}

The approximate beam quality requirements at the entrance to the wiggler for high-power IR FEL operation are discussed in this Appendix. In the FEL, the gain, growth rate, and efficiency are sensitively dependent on the axial velocity spread $\Delta \beta_{z}$ of the electrons. The electron beam quality is measured in terms of emittance and energy spread, the origins of which include (i) temperature of the cathode, (ii) roughness of the emitting surface, (iii) nonuniformity of the emission from the cathode surface, (iv) nonlinearity of electric and magnetic fields, and (v) self-fields. The electron bunch quality requirement for the FEL, i.e., $\Delta \beta_{z}<\lambda / 2 L_{\text {eff }}$, at the entrance to the wiggler can be expressed by the following inequality [36]:

$$
\Delta E_{\text {total }} / E<\lambda_{w}\left(1+a_{w}^{2} / 2\right) /\left(4 L_{\text {eff }}\right),
$$

where the total longitudinal energy spread is

$$
\Delta E_{\text {total }} / E=\varepsilon_{z} /\left(\tau_{b} E\right)+\varepsilon_{n, \perp}^{2} /\left(2 R_{b}^{2}\right)+\pi^{2} a_{w}^{2} R_{b}^{2} /\left(2 \lambda_{w}^{2}\right) .
$$

In Eq. (A2), the first term represents the fractional energy spread due to longitudinal emittance $\varepsilon_{z}$, the second term arises from transverse rms emittance $\varepsilon_{n, \perp}$, and the third term is due to transverse wiggler gradients. The right-hand side of Eq. (A1) is the approximate FEL conversion efficiency, where the effective interaction length, $L_{\text {eff }}$, is the gain length in the case of the amplifier or the wiggler length in the case of the oscillator, $a_{w}$ is the wiggler parameter, and $\lambda_{w}$ is the wiggler period. If the inequality in Eq. (A1) is well satisfied, the spread in the axial velocity of the electrons plays little role in the FEL interaction. As an example, for a MW-class FEL operating in the IR regime, the typical parameters are $\varepsilon_{n, \perp} \approx 15 \mathrm{~mm} \mathrm{mrad}, \varepsilon_{z} \approx 100 \mathrm{keV} \mathrm{psec}, R_{b} \approx 0.3 \mathrm{~mm}$, $\tau_{b} \approx 1 \mathrm{psec}(\mathrm{rms}), E=80 \mathrm{MeV}, a_{w}^{2}=2$, and $\lambda_{w}=$ $3 \mathrm{~cm}$. For these parameters $\varepsilon_{z} /\left(\tau_{b} E\right) \leq 0.13 \%, \varepsilon_{n, \perp}^{2} /$ $\left(2 R_{b}^{2}\right) \approx 0.13 \%, \quad \pi^{2} a_{w}^{2} R_{b}^{2} /\left(2 \lambda_{w}^{2}\right) \approx 0.1 \%$, and $\Delta E_{\text {total }} /$ $E \approx 0.36 \%$. For an amplifier having a gain length of $30 \mathrm{~cm}$ the right-hand side of Eq. (A1) is $\approx 5 \%$ and the inequality is satisfied. For an oscillator the right-hand side of Eq. (A1) is $\sim 1 / 2 N_{w}$, where $N_{w}$ is the number of wiggler periods. For $N_{w}=100$ the inequality is also satisfied.

\section{APPENDIX B: THERMIONIC INJECTORS}

The gridded electron guns used as injectors for rf linacs in several notable FEL experiments, including the Stanford, Boeing, and Novosibirsk FEL injectors, have operated at subharmonics of the main linac frequency, and have used short high voltage pulses synchronized to the phase of the rf to gate the emission and generate electron bunches suitable for injection into the subharmonic cavities. The original Stanford FEL gun [16] was the first to use this approach, producing $\sim 200 \mathrm{pC}$, $\sim 1.3 \mathrm{nsec}$ electron bunches at $11.8 \mathrm{MHz}$ at the entrance of the normally conducting injector section of a $1.3 \mathrm{GHz}$ superconducting linac.

The Boeing FEL gun [14] produced $5.5 \mathrm{nC}$ electron bunches at $\sim 108 \mathrm{MHz}$, the 12th subharmonic of their $1300 \mathrm{MHz}$ linac, using a $\sim 1 \mathrm{kV}, 2$ nsec drive pulse to the grid of an Eimac electron gun. Operating at the 12th subharmonic accomplished two critical goals. First, it permitted the use of a relatively long high voltage pulse to drive the grid of the electron gun; operating the first $\mathrm{rf}$ cavity at $1.3 \mathrm{GHz}$ while filling the same fraction of an $\mathrm{rf}$ period would have required the generation of a $170 \mathrm{psec}$ drive pulse as well as an $\mathrm{rf}$ gun capable of modulating the current on that time scale. Second, it allowed the use of subharmonic bunching to increase the peak current to the level required for the FEL.

However, the problem with subharmonic bunching is that, while increasing peak current, it ultimately limits the average current, since it limits the fraction of rf buckets that are filled with electrons, while the charge in each bucket is limited by space-charge effects. Using a similar approach, the nonsuperconducting Energy Recovery Linac FEL in Novosibirsk [17] used a $300 \mathrm{keV}$ electron gun with $\sim 1$ A of peak current in a $0.5 \mathrm{nsec}$ rms pulse at a maximum repetition rate of $22.5 \mathrm{MHz}$ (eighth subharmonic of the $\sim 180 \mathrm{MHz}$ linac). In this case, the maximum average current was $\sim 20 \mathrm{~mA}$. 
The SPring-8 FEL adopted an innovative approach to producing a high brightness, bunched beam from a thermionic cathode without using a control grid $[18,19]$. The experiment employs a single-crystal $\mathrm{CeB}_{6}$ cathode to produce a high current density $500 \mathrm{keV}$ beam from an electron gun driven by a high-voltage modulator. At the exit to the gun, a beam with a 1 A peak current and $3 \mu$ sec pulse width at a maximum repetition rate of $60 \mathrm{~Hz}$ was generated. A single nanosecond bunch is then generated from each pulse by means of a beam chopper. Thus, more than 99.9\% of the beam is deposited in a beam dump. This gridless approach thus solves the problem of producing a clean, low-emittance electron bunch, but at the cost of depositing most of the beam current in a beam dump, an approach that is inconsistent with high average current applications.

A high average current injector should aim to fill every rf bucket. For a $700 \mathrm{MHz}$ linac with no subharmonic section, a thermionic injector must generate electron bunches of order $100 \mathrm{psec}$ in pulse length at a $700 \mathrm{MHz}$ pulse repetition rate. Moreover, in order to produce an average current of $\sim 1 \mathrm{~A}$, the instantaneous current should be about an order of magnitude higher. For such short bunches and high repetition rate, it is not practical to generate short high-voltage pulses to apply to the grid of an electron gun, and direct rf modulation of the grid is required.

Gridded electron guns are used in inductive output tube (IOT) amplifiers [37-39] and can generate average currents of $\sim 1 \mathrm{~A}$, peak currents of $\sim 5-10 \mathrm{~A}$, cathode-anode voltages of $\sim 35 \mathrm{kV}$, and modulation frequencies $>1 \mathrm{GHz}$. These guns use low-temperature dispenser cathodes combined with grids fabricated from pyrolytic graphite, a polycrystalline form of carbon that will operate at high temperatures and has improved strength and uniformity compared to grids made of tungsten or molybdenum [37]. The grid is typically placed within $\sim 100-300 \mu \mathrm{m}$ of the cathode to permit high-frequency current modulation. In typical operation, these guns are employed to produce electron bunches filling $180^{\circ}$ of rf phase in order to generate the maximum rf power at the drive frequency. However, the degree of beam modulation is not constrained to be $180^{\circ}$ of the rf phase; it depends on the negative bias voltage between the grid and the cathode (typically of order 100-200 V) as well as the rf voltage induced in the cathode-grid gap. Shorter bunches can in principle be obtained by driving the grid circuit simultaneously at the fundamental rf frequency and a higher harmonic.

\section{APPENDIX C: GRID FIELDS AND BEAM EMITTANCE}

For the purpose of estimating the electron beam emittance due to a grid, we consider the 2D cathode, grid, anode configuration shown in Fig. 4. The grid wires form an infinite array located at $z=z_{G}$, are parallel to the $y$ axis and are separated by a distance $2 a$. The radius of the grid wires is small, $r_{o} \ll a, z_{G}$. The grounded cathode is located at $z=0$ and the anode at $z=z_{A}$. The potential on the grid relative to the cathode is denoted by $\Phi_{G}(t)$ and consists of a dc bias and rf component while the anode potential is $\Phi_{A}(t)$. In addition, the field in the anodecathode gap induces a dipole field around each grid wire. The dipole field is localized around the grid wire and has little effect on the electron motion. Although the grid voltage varies at the $\mathrm{rf}$ frequency, it can be considered to be constant for the purpose of this calculation since the electron transit time from the cathode to the grid, $\tau_{G}=$ $z_{G} / \mathrm{v}_{z} \sim 0.1 \mathrm{nsec}$, is small compared to the rf period, $\tau_{\text {rf }} \sim 1.4$ nsec.

\section{Potential for an array of grid wires}

The potential associated with a single grid wire located at $x=0$ and $z=z_{G}$ is

$$
\begin{aligned}
\Phi(x, z)= & -\Phi_{O} \ln \left(\frac{x^{2}+\left(z-z_{G}\right)^{2}}{x^{2}+\left(z+z_{G}\right)^{2}}\right)+\Phi_{A} \frac{z}{z_{A}} \\
& -\Phi_{A} \frac{\left(z-z_{G}\right)}{z_{A}}\left(\frac{r_{o}^{2}}{x^{2}+\left(z-z_{G}\right)^{2}}\right),
\end{aligned}
$$

where $\quad \Phi_{O}=(1 / 2)\left[\Phi_{G}(t)-\Phi_{A}(t)\left(z_{G} / z_{A}\right)\right] / \ln \left(2 z_{G} / r_{o}\right)$. In Eq. (C1) the first term is the potential of a line charge together with its image due to the cathode, the second term is due to the anode potential and the third term is due to the induced dipole charges on the wire. For $r_{o} \ll a, z_{G}$ the potential on the cathode is zero, on the surface of the grid wire it is $\Phi_{G}(t)$ and on the anode it is $\Phi_{A}(t)$. For the typical parameters considered here $\left|\Phi_{G}\right| \approx 100 \mathrm{~V}, \Phi_{A} \approx 40 \mathrm{kV}$, $z_{G} \approx 250 \mu \mathrm{m}, z_{A} \approx 1 \mathrm{~cm}, a \approx 200 \mu \mathrm{m}, r_{o} \approx 30 \mu \mathrm{m}$, and $\ln \left(2 z_{G} / r_{o}\right) \sim 3$.

The fields associated with the single grid wire potential, $\mathbf{E}=-\nabla \Phi$, are

$$
\begin{aligned}
& E_{x}=-2 \Phi_{O}\left(\frac{x}{x^{2}+\left(z+z_{G}\right)^{2}}-\frac{x}{x^{2}+\left(z-z_{G}\right)^{2}}\right)-2 \frac{\Phi_{A} r_{o}^{2}}{z_{A}}\left(\frac{\left(z-z_{G}\right) x}{\left[x^{2}+\left(z-z_{G}\right)^{2}\right]^{2}}\right), \\
& E_{z}=-2 \Phi_{O}\left(\frac{\left(z+z_{G}\right)}{x^{2}+\left(z+z_{G}\right)^{2}}-\frac{\left(z-z_{G}\right)}{x^{2}+\left(z-z_{G}\right)^{2}}\right)-\frac{\Phi_{A}}{z_{A}}+\frac{\Phi_{A} r_{o}^{2}}{z_{A}}\left(\frac{x^{2}-\left(z-z_{G}\right)^{2}}{\left[x^{2}+\left(z-z_{G}\right)^{2}\right]^{2}}\right) .
\end{aligned}
$$


The dipole contribution, last term in Eqs. (C2), is small and can be neglected near the cathode for $z_{G} \gg r_{o}$.

The potential due to an infinite array of grid wires, centered at $z=z_{G}$ and $x= \pm a, \pm 3 a, \pm 5 a, \ldots$ (see Fig. 7), is given by

$$
\begin{aligned}
\Phi(x, z)= & -\sum_{n= \pm 1, \pm 3,}\left[\Phi_{O} \ln \frac{G_{n}(x, z)}{F_{n}(x, z)}+\Phi_{A} \frac{r_{o}^{2}\left(z-z_{G}\right) / z_{A}}{G_{n}(x, z)}\right] \\
& +\Phi_{A} \frac{z}{z_{A}},
\end{aligned}
$$

where $\Phi_{O}=(1 / 2)\left(\Phi_{G}-\Phi_{A} z_{G} / z_{A}\right)\left[\ln \left(2 z_{G} / r_{o}\right)+M_{o}\right]^{-1}$, $F_{n}(x, z)=(x-n a)^{2}+\left(z+z_{G}\right)^{2}, \quad G_{n}(x, z)=(x-$ $n a)^{2}+\left(z-z_{G}\right)^{2}$, and $M_{o}=\sum_{l=1,2,3,}^{\infty} \ln \left[1+z_{G}^{2} /\left(\ell^{2} a^{2}\right)\right]$. The corresponding electric field in the cathode-anode region is

$$
\begin{aligned}
& E_{x}=-2 \sum_{n= \pm 1, \pm 3,}\left[\Phi_{O}\left(\frac{x-n a}{F_{n}(x, z)}-\frac{x-n a}{G_{n}(x, z)}\right)+\frac{\Phi_{A} r_{o}^{2}}{z_{A}}\left(\frac{\left(z-z_{G}\right)(x-n a)}{G_{n}^{2}(x, z)}\right)\right], \\
& E_{z}=-\sum_{n= \pm 1, \pm 3,}\left[2 \Phi_{O}\left(\frac{z+z_{G}}{F_{n}(x, z)}-\frac{z-z_{G}}{G_{n}(x, z)}\right)-\frac{\Phi_{A} r_{o}^{2}}{z_{A}}\left(\frac{(x-n a)^{2}-\left(z-z_{G}\right)^{2}}{G_{n}^{2}(x, z)}\right)\right]-\frac{\Phi_{A}}{z_{A}} .
\end{aligned}
$$

The axial electric field at the surface of the cathode in the absence of space-charge is

$$
E_{z}(x, z=0)=-\Phi_{A} / z_{A}-\mu(x)\left(\Phi_{G} / z_{G}-\Phi_{A} / z_{A}\right),
$$

where the dipole term has been neglected and

$$
\mu(x)=\left(\frac{2}{\ln \left(2 z_{G} / r_{o}\right)+M_{o}}\right) \sum_{n= \pm 1, \pm 3,} \frac{z_{G}^{2}}{(x-n a)^{2}+z_{G}^{2}}<1
$$

is a shielding parameter. The grid wires partially shield the electric fields associated with the anode potentials. The electron current is turned off when the grid potential is sufficiently negative so that $E_{z}>0$ on the cathode, that is, no current flows when

$$
\Phi_{G}<-\left(\frac{1-\mu}{\mu}\right) \frac{z_{G}}{z_{A}} \Phi_{A} .
$$

\section{Grid emittance estimate}

The emittance growth due to the grid can be estimated by calculating the rms angular deflection of the electrons as they propagate from the cathode, through the grid region, to the anode (see Fig. 7). The rms normalized emittance $(x$ direction) is $\varepsilon_{n, x}=\gamma \beta_{z}\left(\left\langle x^{2}\right\rangle\left\langle\left(x^{\prime}\right)^{2}\right\rangle-\left\langle x x^{\prime}\right\rangle^{2}\right)^{1 / 2}$, where \langle\rangle denotes an average over particles, $\gamma \approx 1$ is the relativistic factor, $\beta_{z}=v_{z} / c, v_{z}$ is the axial beam velocity, and $x^{\prime}=$ $\partial x / \partial z$. The normalized emittance is given by $\varepsilon_{n, x} \approx$ $\gamma \beta_{z} R_{b}\langle\Delta \theta\rangle$, where $R_{b}$ is the rms beam radius and $\langle\Delta \theta\rangle=$ $\left\langle v_{x} / v_{z}\right\rangle$ is the rms deflection angle. The normalized grid emittance can therefore be written as $\varepsilon_{n, x} \approx R_{b}\left\langle v_{x} / c\right\rangle$. In the absence of self-fields the electron deflection velocity in the $x$ direction is given by $\partial v_{x} / \partial z=(q / m) E_{x} / v_{z}$, where the transverse electric field near the $z$ axis, $|x|<a$, can be approximated by $E_{x} \approx-x \partial E_{z} / \partial z$, where $E_{x}$ and $E_{z}$ are the electric field components associated with the dc bias and rf fields.
The transverse deflection velocity at $z=2 z_{G}$ is approximately given by

$$
v_{x} \approx-\frac{q}{m} \int_{0}^{2 z_{G}} \frac{x}{v_{z}} \frac{\partial E_{z}}{\partial z} d z \approx-\frac{q x}{m\left\langle v_{z}\right\rangle} \Delta E_{z},
$$

where $\Delta E_{z}=E_{z}\left(x, 2 z_{G}\right)-E_{z}(x, 0)$. It is assumed that the transverse electron position changed little from its initial position and $\left\langle v_{z}\right\rangle$ is to be evaluated at $z=2 z_{G}$. Beyond $z \sim 2 z_{G}$ the transverse velocity is approximately constant. For $r_{o} \ll a, z_{G}$, the effect of the dipole field part of the potential on the average electron deflection can be neglected.

To obtain an estimate for the emittance, we consider a single wire centered at $z=z_{G}$ and $x=0$, making use of the potential and fields given by Eqs. (C1) and (C2). The axial field difference, using Eq. (C2), is

$$
\Delta E_{z} \approx \frac{4}{3} \mu_{1}(x)\left(\frac{\Phi_{G}}{z_{G}}-\frac{\Phi_{A}}{z_{A}}\right)
$$

where

$$
\mu_{1}(x)=\left(\frac{2}{\ln \left(2 z_{G} / r_{o}\right)}\right) \frac{z_{G}^{2}}{\left(x^{2}+z_{G}^{2}\right)}
$$

is the shielding parameter associated with a single grid wire. The normalized transverse velocity at $z=2 z_{G}$ is

$$
\beta_{x} \approx-\frac{4}{3} \frac{q x}{m c^{2}\left\langle\beta_{\mathrm{z}}\right\rangle} \mu_{1}(x)\left(\frac{\Phi_{G}}{z_{G}}-\frac{\Phi_{A}}{z_{A}}\right) .
$$

To approximate the average deflection due to an array of grid wires we take $x \approx \pm a$. In this case the axial velocity is $\left\langle\beta_{z}\right\rangle \approx\left[2 q \Phi(x, z) / m c^{2}\right]^{1 / 2}$ for $\langle\Delta \theta\rangle \ll 1$, where $\Phi\left(x, 2 z_{G}\right) \approx 2\left(z_{G} / z_{A}\right) \Phi_{A}$. The grid emittance is therefore given by

$$
\varepsilon_{n, \text { grid }} \approx R_{b}\left\langle\beta_{x}\right\rangle \approx \frac{2 \mu_{1}(a)}{3}\left(\frac{a}{z_{G}}\right) R_{b} \frac{\left|\bar{\Phi}_{G}-\left(z_{G} / z_{A}\right) \bar{\Phi}_{A}\right|}{\left(z_{G} / z_{A}\right)^{1 / 2} \bar{\Phi}_{A}^{1 / 2}},
$$


where $\bar{\Phi}_{G}=|q| \Phi_{G} /\left(m c^{2}\right)$ and $\bar{\Phi}_{A}=|q| \Phi_{A} /\left(m c^{2}\right)$. The electric field on the cathode for a single grid wire is $E_{z}(x, 0)=-\Phi_{A} / z_{A}-\mu_{1}(x)\left(\Phi_{G} / z_{G}-\Phi_{A} / z_{A}\right)$. Using typical values for the parameters, we find that $\left\langle\beta_{z}\right\rangle \sim 2 \times$ $10^{-2}$ and the normalized emittance due to the grid is $\varepsilon_{n \text {, grid }} \sim 5-15 \mathrm{~mm} \mathrm{mrad}$.

A number of approximations were employed in obtaining the grid emittance. More accurate estimates for the grid emittance, which include self-field effects, are obtained using the PIC codes TURBOWAVE and PARMELA and are presented in Sec. III.

[1] G. R. Neil, C. L. Bohn, S. V. Benson, G. Biallas, D. Douglas, H. F. Dylla, R. Evans, J. Fugitt, A. Grippo, J. Gubeli, R. Hill, K. Jordan, G. A. Krafft, R. Li, L. Merminga, P. Piot, J. Preble, M. Shinn, T. Siggins, R. Walker, and B. Yunn, Phys. Rev. Lett. 84, 662 (2000).

[2] "Free Electron Laser High Brightness, High Average Current Injector" report to the NAVY HEL Program Office (PMS-405) \& Office of Naval Research,' P. O'Shea, Panel Chairman, 2002.

[3] A. Todd, Nucl. Instrum. Methods Phys. Res., Sect. A 557, 36 (2006).

[4] I. Ben-Zvi and I. V. Bazarov, Nucl. Instrum. Methods Phys. Res., Sect. A 557, 337 (2006).

[5] W. B. Colson, J. Blau, and R. L. Armstead, Nucl. Instrum. Methods Phys. Res., Sect. A 507, 48 (2003).

[6] D. C. Nguyen and H. P. Freund, Nucl. Instrum. Methods Phys. Res., Sect. A 507, 120 (2003).

[7] P. Sprangle, B. Hafizi, and J. R. Penano, IEEE J. Quantum Electron. 40, 1739 (2004).

[8] I. Ben-Zvi, D. Kayran, and V. Litvinenko, in Proceedings of the International FEL Conference, Stanford, CA, 2005, p. 232, http://www.jacow.org.

[9] P. Sprangle, J. Penano, B. Hafizi, and I. Ben-Zvi, IEEE J. Quantum Electron. 46, 1135 (2010).

[10] D. C. Nguyen et al., Nucl. Instrum. Methods Phys. Res., Sect. A 528, 71 (2004).

[11] S. Benson et al., in Proceedings of the 2004 FEL Conference (Comitato Conferenze ELETTRA, Trieste, Italy, 2004), p. 229.

[12] K. L. Jensen et al., J. Appl. Phys. 102, 074902 (2007).

[13] C. Hernandez-Garcia, T. Siggins, S. Benson, D. Bullard, H. F. Dylla, K. Jordan, C. Murray, G. R. Neil, M. Shinn, and R. Walker, in Proceedings of the 21st Particle Accelerator Conference, Knoxville, 2005 (IEEE, Piscataway, NJ, 2005), p. 3117; C. Hernandez-Garcia, P. G. O'Shea, and M.L. Stutzman, Phys. Today 61, No. 2, 44 (2008).

[14] A. Yeremian et al., in Proceedings of the Particle Accelerator Conference, 1989. Accelerator Science and Technology, Chicago, IL, USA (IEEE, Piscataway, NJ, 1989), pp. 657-659.

[15] J. M. J. Madey, G. J. Ramian, and T. I. Smith, IEEE Trans. Nucl. Sci. 27, 999 (1980).
[16] T.I. Smith, Physics of Quantum Electronics, edited by S. F. Jacobs et al. (Addison-Wesley, Reading, MA, 1982), Vol. 8, pp. 77-87.

[17] V.P. Bolotin et al., in Proceedings of the 2004 FEL Conference (Comitato Conferenze ELETTRA, Trieste, Italy, 2004), pp. 226-228; S. V. Miginsky et al., in Proceedings of the 2nd Annual Asian Particle Accelerator Conference, Beijing, China, 2001, pp. 82-84, http://www.jacow.org.

[18] K. Togawa et al., Phys. Rev. ST Accel. Beams 10, 020703 (2007).

[19] T. Shintake et al., Phys. Rev. ST Accel. Beams 12, 070701 (2009).

[20] R. J. Bakker, C. A. J. van der Geer, A. F. G. vanderMeer, P.W. van Amersfoort, W. A. Gillespie, and G. Saxon, Nucl. Instrum. Methods Phys. Res., Sect. A 307, 543 (1991).

[21] D. F. Gordon, W. B. Mori, and T. M. Antonsen, Jr., IEEE Trans. Plasma Sci. 28, 1135 (2000); D. F. Gordon, IEEE Trans. Plasma Sci. 35, 1486 (2007).

[22] J. H. Billen, PARMELA Documentation, Los Alamos Report No. LA-UR-96-1835, revised 2005.

[23] A. S. Gilmore, Principles of Travelling Wave Tubes (Artech House, Boston, MA, 1994).

[24] P. Frigola et al., in Proceedings of the 20th Particle Accelerator Conference, Portland, OR, 2003 (IEEE, New York, 2003), http://www.jacow.org.

[25] R. Thomas, J. Gibson, G. Haas, and R. H. Abrams, Jr., IEEE Trans. Electron Devices 37, 850 (1990).

[26] M. Reiser, Theory and Design of Charged Particle Beams (Wiley, New York, 1994).

[27] T. Wangler, RF Linear Accelerators (Wiley, New York, 1998).

[28] R. C. Davidson, Physics of Nonneutral Plasmas (AddisonWesley, Redwood, CA, 1990).

[29] R. Vaughan, IEEE Trans. Electron Devices 33, 1925 (1986).

[30] S. H. Gold and G. S. Nusinovich, Rev. Sci. Instrum. 68, 3945 (1997).

[31] Y. Y. Lau, Y. Liu, and R. K. Parker, Phys. Plasmas 1, 2082 (1994).

[32] J.D. Jarvis et al., in Proceedings of the International FEL Conference, Gyeongju, Korea, 2008, p. 477, http:// www.jacow.org.

[33] J.D. Lawson, The Physics of Charged-Particle Beams (Oxford University Press, Oxford, UK, 1988).

[34] L. Serafini and J. B. Rosenzweig, Phys. Rev. E 55, 7565 (1997).

[35] Communications \& Power Industries (CPI) (private communication).

[36] C. W. Roberson and P. Sprangle, Phys. Fluids B 1, 3 (1989).

[37] E. G. Zaidman and M. A. Kodis, IEEE Trans. Electron Devices 38, 2221 (1991).

[38] C. Beard, Nucl. Instrum. Methods Phys. Res., Sect. A 557, 276 (2006).

[39] D. H. Priest and M. B. Shrader, Proc. IEEE 70, 1318 (1982). 